\title{
Carbon dust in the evolved born-again planetary nebulae A 30 and A 78
}

\author{
J.A. Toalál ${ }^{1 \star}$, P. Jiménez-Hernández ${ }^{1}$, J.B. Rodríguez-González ${ }^{1}$, S. Estrada-Dorado ${ }^{1}$, M.A. Guerrero ${ }^{2}$ \\ V.M.A. Gómez-González ${ }^{1}$, G. Ramos-Larios ${ }^{3,4}$, D.A. García-Hernández ${ }^{5,6}$ and H. Todt $^{7}$ \\ ${ }^{1}$ Instituto de Radioastronomía y Astrofísica, UNAM Campus Morelia, Apartado postal 3-72, 58090 Morelia, Mich., Mexico \\ ${ }^{2}$ Instituto de Astrofísica de Andalucía, IAA-CSIC, Glorieta de la Astronomía S/N, Granada 18008, Spain \\ ${ }^{3}$ CUCEI, Universidad de Guadalajara, Blvd. Marcelino García Barragán 1421, 44430, Guadalajara, Jalisco, Mexico \\ ${ }^{4}$ Instituto de Astronomía y Meteorología, Dpto. de Física,CUCEI, Av. Vallarta 2602, 44130, Guadalajara, Jalisco, Mexico \\ ${ }^{5}$ Instituto de Astrofísica de Canarias, C/Via Lactea s/n, E-38200 La Laguna, Spain \\ ${ }^{6}$ Universidad de La Laguna (ULL), Departamento de Astrofísica, E-38206 La Laguna, Tenerife, Spain \\ ${ }^{7}$ Institute for Physics and Astronomy, Universität Potsdam, D-14476 Potsdam, Germany
}

26 February 2021

\section{ABSTRACT}

We present an infrared (IR) characterization of the born-again planetary nebulae (PNe) A 30 and A 78 using IR images and spectra. We demonstrate that the carbon-rich dust in A 30 and A 78 is spatially coincident with the H-poor ejecta and coexists with hot X-ray-emitting gas up to distances of $50^{\prime \prime}$ from the central stars (CSPNs). Dust forms immediately after the born-again event and survives for $1000 \mathrm{yr}$ in the harsh environment around the CSPN as it is destroyed and pushed away by radiation pressure and dragged by hydrodynamical effects. Spitzer IRS spectral maps showed that the broad spectral features at 6.4 and $8.0 \mu \mathrm{m}$, attributed to amorphous carbon formed in H-deficient environments, are associated with the disrupted disk around their CSPN, providing an optimal environment for charge exchange reactions with the stellar wind that produces the soft X-ray emission of these sources. Nebular and dust properties are modeled for A 30 with cLoudy taking into account different carbonaceous dust species. Our models predict dust temperatures in the 40-230 K range, five times lower than predicted by previous works. Gas and dust masses for the born-again ejecta in A 30 are estimated to be $M_{\text {gas }}=\left(4.41_{-0.14}^{+0.55}\right) \times 10^{-3} \mathrm{M}_{\odot}$ and $M_{\text {dust }}=\left(3.20_{-2.06}^{+3.21}\right) \times 10^{-3} \mathrm{M}_{\odot}$, which can be used to estimate a total ejected mass and mass-loss rate for the born-again event of $\left(7.61_{-2.20}^{+3.76}\right) \times 10^{-3} \mathrm{M}_{\odot}$ and $\dot{M}=[5-60] \times 10^{-5} \mathrm{M}_{\odot} \mathrm{yr}^{-1}$, respectively. Taking into account the carbon trapped into dust grains, we estimate that the $\mathrm{C} / \mathrm{O}$ mass ratio of the $\mathrm{H}$-poor ejecta of A 30 is larger than 1, which favors the very late thermal pulse model over the alternate hypothesis of a nova-like event.

Key words: ISM: molecules — stars: evolution — stars: winds, outflows — planetary nebulae: individual (A 30, A 58, A 78) - infrared: ISM

\section{INTRODUCTION}

Born-again planetary nebulae $(\mathrm{PNe})$ represent a very specific case in the evolution of low- and intermediate-mass stars $\left(M_{\mathrm{i}} \lesssim 1-8 \mathrm{M}_{\odot}\right)$. By the late 1970s and early 1980s, theoretical works proposed that the H-rich outer layer of a central star of a PN (CSPN) could build a shell of helium with the critical mass to ignite into carbon and oxygen (see Schönberner 1979; Iben et al. 1983), producing what is known today as a very late thermal pulse (VLTP; e.g., Herwig et al. 1999). According to this process, an explosive event would eject newly processed, metal-rich material inside the old hydrogen-rich PN while leaving the central star intact. Models such as those presented by Miller Bertolami et al. (2006) predict that for a short period of time ( 20-100 yr), the star returns to the asymptotic giant branch (AGB) region of the HR diagram to finally evolve into a second white dwarf phase developing a new fast stellar wind and increasing its ionizing photon flux. The result is what it is commonly know as a born-again PN. Although the number of reported born-again PNe

\footnotetext{
^ E-mail:j.toala@irya.unam.mx
}

is small (see Guerrero et al. 2018; Gvaramadze et al. 2020, for the latest discoveries), they give the opportunity to study complex effects which take place in human-time scales.

The most studied object of this class is the one known as Sakurai's Object (a.k.a. V 4334 Sgr), which experienced an outburst in 1996 (Nakano et al. 1996). Sakurai's Object is surrounded by a hydrogenrich outer nebula with an angular diameter of $\sim 40^{\prime \prime}$ (e.g., Duerbeck et al. 1996; Pollacco 1999), but early studies showed a considerable change in composition consistent with the ingestion of the hydrogen shell and the generation of $s$-process elements (Asplund et al. 1997). Within $20 \mathrm{yr}$, the CSPN of Sakurai's Object and its ejecta have exhibited dramatic changes (e.g., Evans et al. 2020). The CSPN varied its flux from $K \sim 11.4$ mag in 1996 February to $K \sim 25$ mag by 1999 , becoming obscured by dust and no longer detectable in optical wavelengths (Duerbeck et al. 2000). Very Large Array (VLA) observations pointed at the reionization of the central core of Sakurai's Object by 2004 (see van Hoof et al. 2007, and references therein), yet the dust shell was still cooling by 2005 at temperatures $\sim 200 \mathrm{~K}$ according to Spitzer IRS observations (Evans et al. 2006). The ejecta has kept expanding since then, decreasing its optical thickness as revealed 
by the increase in magnitude up to $K_{\mathrm{S}}=13.8 \mathrm{mag}$ by 2014 June (Hinkle \& Joyce 2015), until it has been finally detected in near-IR observations a bipolar ejecta being blown by the current increase of the stellar wind from the CSPN of Sakurai's Object. Sub-millimeter observations report that the hydrogen-poor ejecta is currently moving at velocities $\gtrsim 100 \mathrm{~km} \mathrm{~s}^{-1}$ (see, e.g., Tafoya et al. 2017).

The detailed morphology and angular expansion of the more evolved born-again PNe A 30, A 58 and A 78 have been investigated using Hubble Space Telescope (HST) observations (Clayton et al. 2013; Fang et al. 2014). Similarly to Sakurai's Object, these three objects exhibit a nearly spherical outer hydrogen-rich PNe, but their VLTP ejecta are rather asymmetric. A 58, which experienced a VLTP around $100 \mathrm{yr}$ ago, exhibits the clear presence of a hydrogen-deficient disk which is being disrupted by the current fast wind $\left(v_{\infty} \approx 2500\right.$ $\mathrm{km} \mathrm{s}^{-1}$; Clayton et al. 2006) and the increased UV flux from its CSPN (Hinkle et al. 2008). A 30 and A 78 are the oldest objects of this class with a time-lapse since the VLTP event $\lesssim 1000$ yr (Fang et al. 2014). Hydrogen-poor structures were discovered around their CSPNe (see Fig. 1 left panels) by Jacoby (1979) and subsequent high resolution HST observations (Borkowski et al. 1993, 1995) revealed that these seem to be arranged in disrupted disks and bipolar outflows orthogonal to them (see Fig. 2 and 3 upper left panels; e.g, Meaburn et al. 1998; Fang et al. 2014, and references therein). These morphologies have been used to argue that their CSPNe might be binary systems. Indeed, Jacoby et al. (2020) has recently reported periodic variations in the light curve of the CSPN of A 30, which they attribute to the presence of a binary system.

\subsection{Dust formation in born-again PNe}

As a consequence of the VLTP, the CSPN moves back to the AGB region of the HR diagram for a short period of time. The significant reduction of the effective temperature $\left(T_{\text {eff }}\right)$ allows the formation of dust from the $\mathrm{H}$-poor, $\mathrm{C}$-enhanced material that will be injected into the old PN. Evans et al. (2020) showed the dramatic evolution of the dust and molecules in Sakurai's Object in a time period of only $20 \mathrm{yr}$, when the dust in its born-again ejecta cooled down from more than $1000 \mathrm{~K}$ to $\sim 180 \mathrm{~K}$ and its mass increased from $10^{-10} \mathrm{M}_{\odot}$ to $10^{-5} \mathrm{M}_{\odot}$. On the other hand, the $100 \mathrm{yr}$ old ejecta in A 58 has been reported to harbor dust with a range of temperatures, $\lesssim 10^{-5} \mathrm{M}_{\odot}$ of warm dust with temperatures in the $200-800 \mathrm{~K}$ range and $2 \times 10^{-3}$ $\mathrm{M}_{\odot}$ of cold dust with a temperature of $75 \mathrm{~K}$ (Clayton et al. 2013). The latter is in the range of the dust mass of $8 \times 10^{-3} \mathrm{M}_{\odot}$ estimated by Koller \& Kimeswenger (2001).

The exceptional dust enrichment of A 30 and A 78 compared to other PNe was first noted in the analysis of IR photometric observations in the 2.2-22 $\mu \mathrm{m}$ wavelength range of $113 \mathrm{PNe}$ presented by Cohen, \& Barlow (1974). The photometry of A 30 suggested that most of the IR emission did not arise from the CSPN, but from an extended $\sim 25-30^{\prime \prime}$ in diameter region within the main nebula (see also Moseley 1980). This conclusion was supported by subsequent work presented by Cohen et al. (1977) who studied optical, near- and mid-IR observations of A 30 and A 78 and concluded that thermal emission from dust should be extended with dust present up to distances as far as $\gtrsim 10-30^{\prime \prime}$ from their CSPNe. The first ever direct IR image of any born-again PN presented by Dinerstein $\&$ Lester (1984) corroborated the suggestion of previous works that the dust distribution is clumpy. Moreover, their $K$ and $N$ near-IR images of the central region of A 30 suggested that dust was mainly distributed along a disrupted disk, although the spatial resolution of the observations was not optimal.

The first high-resolution near-IR $K$-band image of A 30 resolved in great detail the hot dust of its inner region, which is spatially associated with the disrupted disk around the CSPN (Borkowski et al. 1994). These authors used all available IR photometry covering the wavelength range from 1.2 to $25 \mu \mathrm{m}$ (Pottasch et al. 1984; Leene \& Pottasch 1988) and produced models of thermally-emitting carbon rich dust. The smallest size of the dust grains in these models had to be $0.0007 \mu \mathrm{m}$ in order to fit the IR spectral energy distribution (SED) around $1-5 \mu \mathrm{m}$, with a complete size distribution of $0.0007-0.25 \mu \mathrm{m}$. Furthermore, the presence of graphite and polycyclic aromatic hydrocarbons (PAHs) in the dust of A 30 was rejected and calculations performed using only amorphous carbon grains. The final model presented by Borkowski et al. (1994) includes contribution from two concentric shells, one accounting for the disrupted disk and jets and another for the extended clumpy distribution around A 30 (see Fig. 1 top left panel). This model predicts a dust mass of $3.6 \times 10^{-5} \mathrm{M}_{\odot}$ for the inner shell and about 60 times more for the outer shell, $2.2 \times 10^{-3} \mathrm{M}_{\odot}$.

Recently, Muthumariappan \& Parthasarathy (2020) presented a statistical analysis of the IR properties of the PNe with Wolf-Rayettype CSPN compiled by Weidmann \& Gamen (2011) using photometric measurements from the Two Micron All Sky Survey (2MASS), Wide-field Infrared Survey (WISE), Infrared Astronomical Satellite (IRAS), and Akari. This study computed the dust colour temperatures, dust masses, and dust-to-gas mass ratios, among other quantities of these PNe. They report a dust mass of $10^{-4} \mathrm{M}_{\odot}$ for A 30, with a dust-to-gas ratio $>30$. No values were reported for A 78 .

In this paper we present the analysis of IR images and spectra of A 30 and A 78. The images reveal in great detail the spatial distribution of multiple dust components in these $\mathrm{PNe}$, whereas the spectra inform about their mid-IR spectral properties. A model of the gas and dust tailored specifically to A 30 has been used to estimate its total mass ejected during the VLTP event. This paper is organized as follows. The observations are described in Section 2. The distribution of dust and its spectral properties are presented in Sections 3 and 4 , respectively. Our dust model and its predictions are introduced in Section 5. We discuss our results in Section 6 and present our summary and conclusions in Section 7.

\section{OBSERVATIONS}

IR observations of A 30 and A 78 were obtained from different telescopes. Archival Spitzer IRS and IRAC observations as well as WISE images were obtained from the NASA/IPAC Infrared Science Archive ${ }^{1}$. A 30 was observed by Spitzer on 2007 November 25 and December 11 in the staring mode. The IRAC and IRS observations correspond to Program ID. 40115 (PI:G. Fazio; AORKey: 21967616, 21967872 and 21968128). The Spitzer IRS observations of A 78 used here correspond to Program ID. 3362 (PI: A. Evans; AORKey: 10839808) and were obtained on 2004 November 6, 14 and 17 in mapping mode. For comparison and discussion, we also retrieved the Spitzer IRS observations of A 58 obtained on 2004 October 23 as part of the Program ID. 3362 (PI: A. Evans: AORKey: 10838272 and 10841600).

The low-resolution IRS observations were obtained with the shortlow (SL) and long-low (LL) modules, covering together the wavelength range $\lambda=5-38 \mu \mathrm{m}$. The IRS data also include observations obtained with the high-resolution modules short-high (SH) and long-

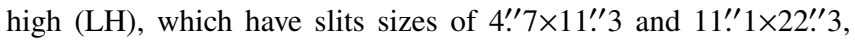

\footnotetext{
1 https://irsa.ipac.caltech.edu/frontpage/
} 

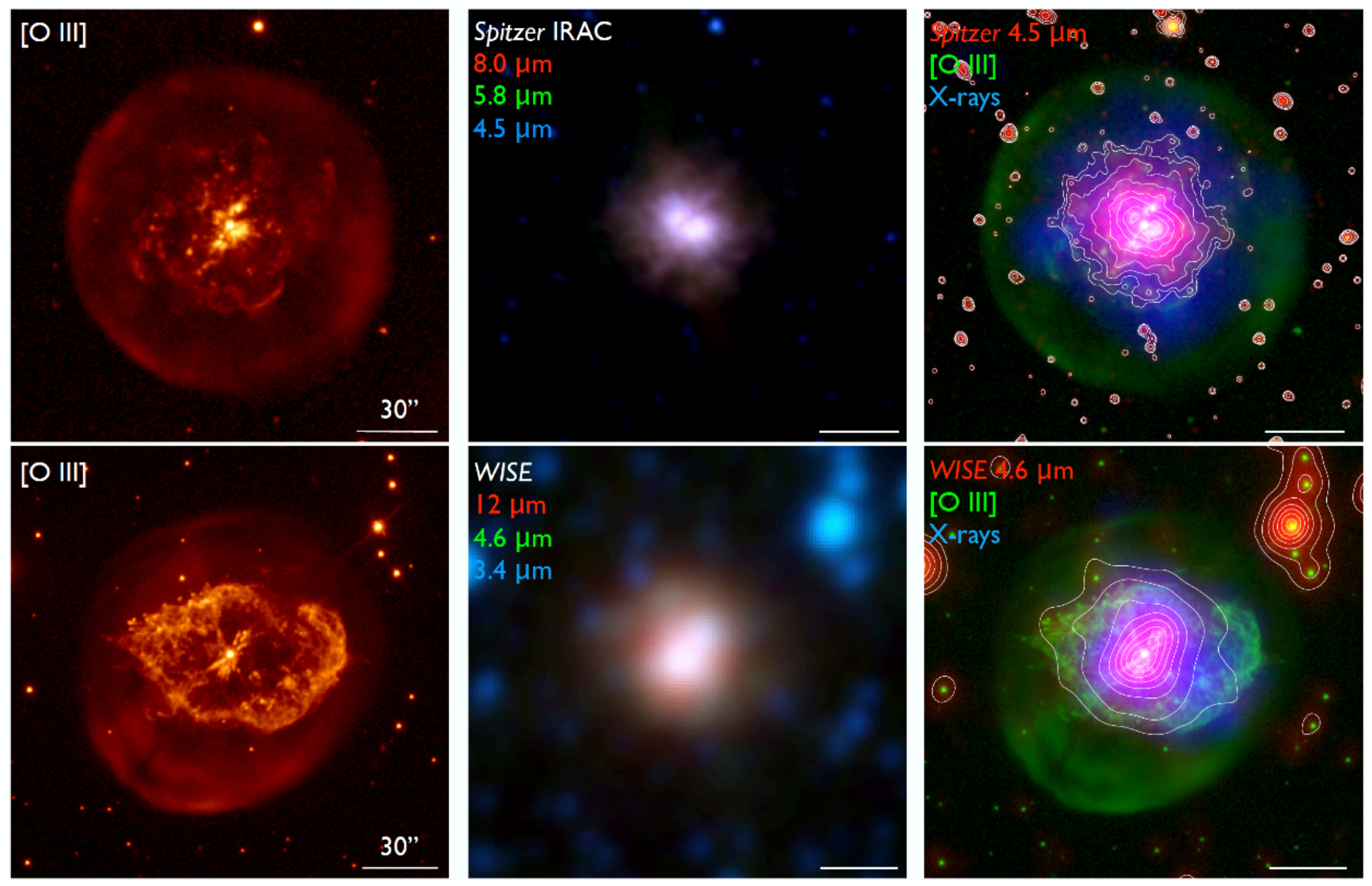

Figure 1. Comparison between the IR, optical and X-ray emission of A 30 (top panels) and A 78 (bottom panels). The left panels show optical [O III] images obtained at the Kitt Peak National Observatory Mayall telescope for A 30 and the Nordic Optical Telescope (NOT) for A 78, the middle panels show colourcomposite IR images, and the right panels show colour-composite images combining IR (red), optical [O III] (green) and XMM-Newton soft X-ray (0.3-0.5 keV; blue) images. To facilitate the comparison we show the IR contours. In all images North is up, East to the left.

respectively, positioned at the CSPNe. The spectral range covered by the high-resolution IRS observations is $\lambda=10-37 \mu \mathrm{m}$. All IRS observations were processed using the CUbe Builder for IRS Spectra Maps (Smith et al. 2007). We note that the low-resolution spectra of A 30 and A 78 were analyzed by García-Hernández et al. (2013) in comparison with the extremely $\mathrm{H}$-deficient $\mathrm{R}$ Coronae Borealis (RCB) stars to discuss the C-rich origins of these sources. Those authors were mainly interested in the broad dust features below $10 \mu \mathrm{m}$ (the RCB stars generally display featureless spectra at longer wavelengths) and did not analyze the high-resolution Spitzer spectra.

Near-IR images of A 30 and A 78 were obtained with the NOTCam at the Nordic Optical Telescope (NOT). The observations were obtained on 2014 April 15-16 and August 9-10 through $J$ $\left(\lambda_{\mathrm{c}}=1.25 \mu \mathrm{m}, \Delta \lambda=0.16 \mu \mathrm{m}\right), H\left(\lambda_{\mathrm{c}}=1.63 \mu \mathrm{m}, \Delta \lambda=0.29 \mu \mathrm{m}\right)$, and $K_{\mathrm{s}}\left(\lambda_{\mathrm{c}}=2.14 \mu \mathrm{m}, \Delta \lambda=0.28 \mu \mathrm{m}\right)$ filters. The total $J, H$ and $K$ exposure times for A 30 were 120, 180 and 240 s and 144, 144 and $192 \mathrm{~s}$ for A 78. All near-IR observations were reduced following standard IRAF routines (Tody 1993). The resultant $J, H$ and $K$ images of A 30 and A 78 are presented in the top panels of Figures 2 and 3, respectively.

Mid-IR Gran Telescopio de Canarias (GTC) CanariCam observations of A 78 were also obtained on 2014 Dec 12-13 and 2015 August 3 (PI: M.A. Guerrero). Images were acquired using the Si3-9.8 $\left(\lambda_{\mathrm{c}}=9.8 \mu \mathrm{m}, \Delta \lambda=1.0 \mu \mathrm{m}\right)$, NeII-12.8 $\left(\lambda_{\mathrm{c}}=12.8 \mu \mathrm{m}, \Delta \lambda=0.2 \mu \mathrm{m}\right)$, and NeII_ref2-13.1 $\left(\lambda_{\mathrm{c}}=13.1 \mu \mathrm{m}, \Delta \lambda=0.2 \mu \mathrm{m}\right)$ filters ${ }^{2}$ with total exposure times of $1500 \mathrm{~s}$ on each filter. The CanariCam images were reduced following the REDCAN reduction pipeline (González-Martín et al. 2013). It is important to notice that the CanariCam observations cover a field of view of $26^{\prime \prime} \times 19^{\prime \prime}$ and only partially map the inner region of A 78 (see bottom panels in Fig. 3).

For comparison with the IR images and further discussion we also include in our analysis the optical (ground-based and HST) [O III] and X-ray XMM-Newton EPIC images of A 30 and A 78 published by our team in previous works (see Fang et al. 2014; Guerrero et al. 2012; Toalá et al. 2015). The [O III] narrow-band images of A 30 and A 78 are presented in the left panels of Figure 1.

\section{SPATIAL DISTRIBUTION OF DUST}

Figure 1 shows the IR images of A 30 and A 78 in comparison with their optical [O III] images. The Spitzer IRAC and WISE images of A 30 and A 78 unveil in unprecedented detail the distribution of the hot dust around their CSPN. The dust extends up to distances

\footnotetext{
${ }^{2}$ For a complete list of GTC CanariCam filters and their details see http://www.gtc.iac.es/instruments/canaricam/canaricam. php\#Filters.
} 


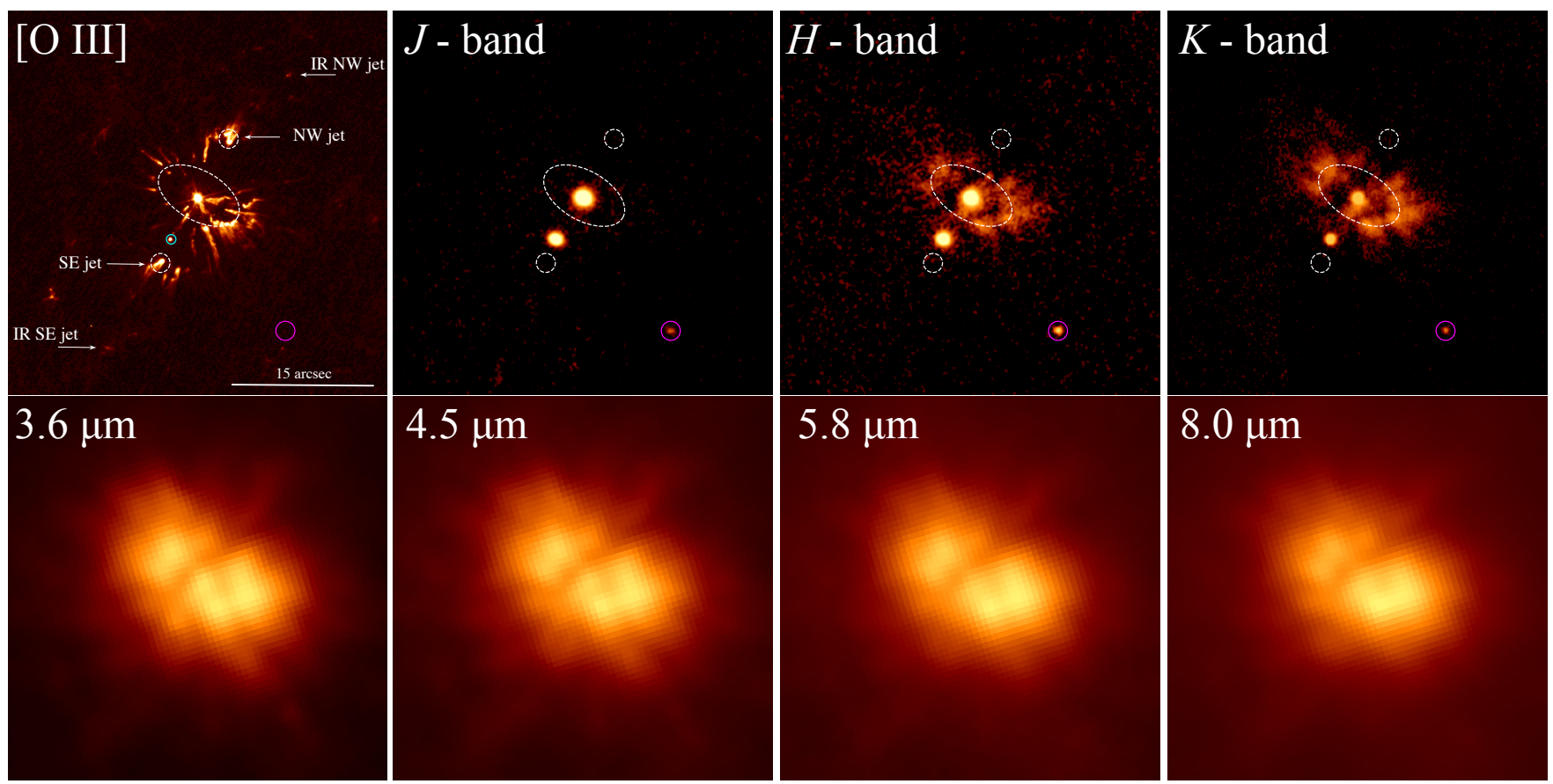

Figure 2. The inner region of A 30 as seen in different wavelengths. The top panels show the $H S T$ [O III] narrow-band (leftmost panel) and NOTCam $J$, $H$ and $K$ images, whereas the bottom panels show Spitzer IRAC 3.6, 4.5, 5.8 and $8.0 \mu$ m images. The disrupted disk extension is shown with a dashed-line ellipse while the positions of the jet features are marked by dashed-line circles in the top panels. A background star is shown with cyan circle. The position of a clump that appears in the NOTCam images is shown with a magenta circle. All panels correspond to the same FoV. North is up, East to the left.

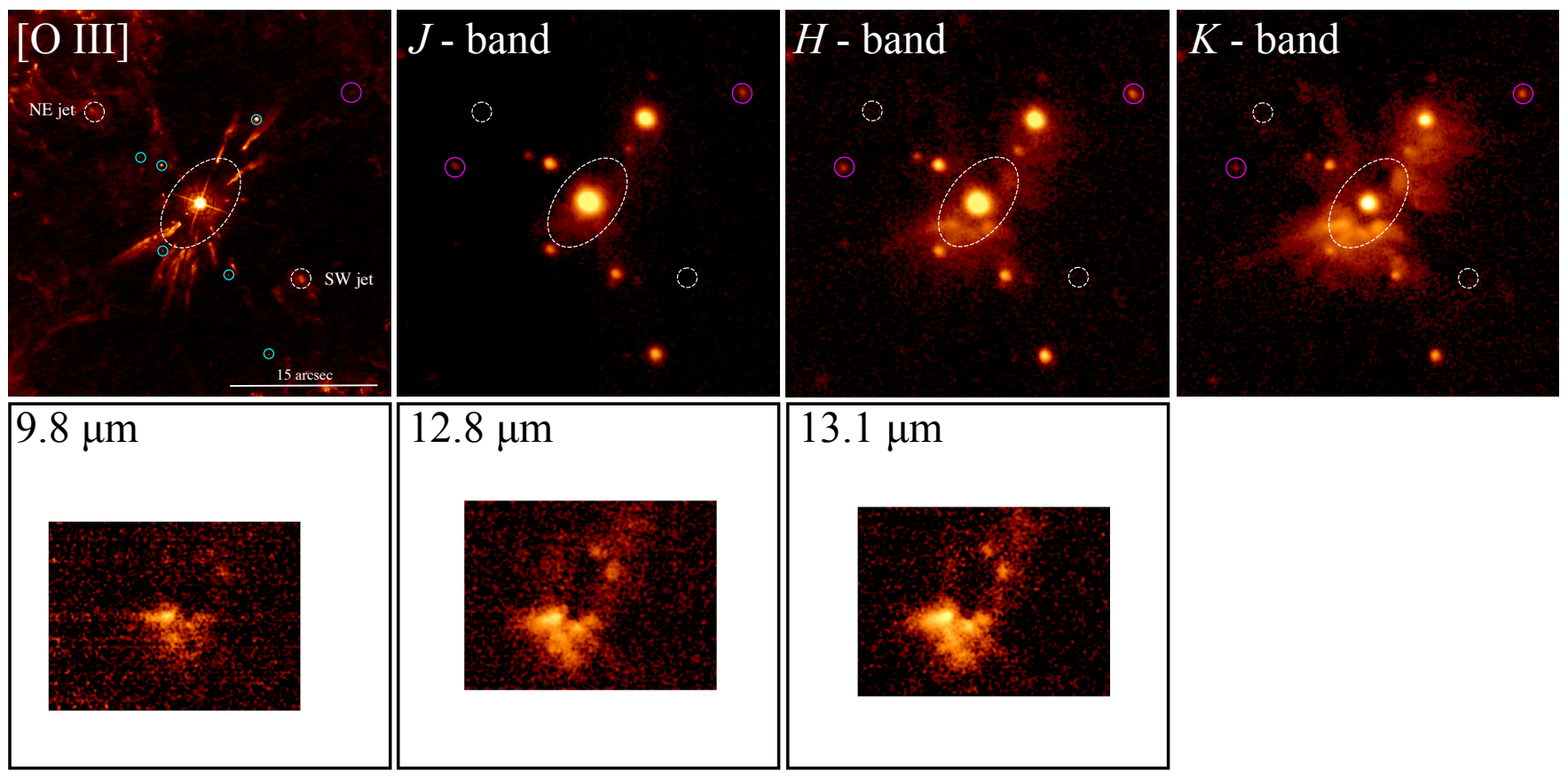

Figure 3. The inner region of A 78 as seen in different wavelengths. The top panels show the $H S T$ [O III] narrow-band (leftmost panel) and NOTCam $J, H$ and $K$ images, whereas the bottom panels show GTC CanariCam images in different bands. The disk extension is shown with a dashed-line ellipse while the positions of the jet features are marked by dashed-line circles in the top panels. Background stars are shown with cyan circles. The position of clumps that appear in the NOTCam images are shown with a magenta circles. All panels correspond to the same FoV, but note that the CanariCam observations only map a region of $26^{\prime \prime} \times 19^{\prime \prime}$ of the central area of A 78 . North is up, East to the left. 

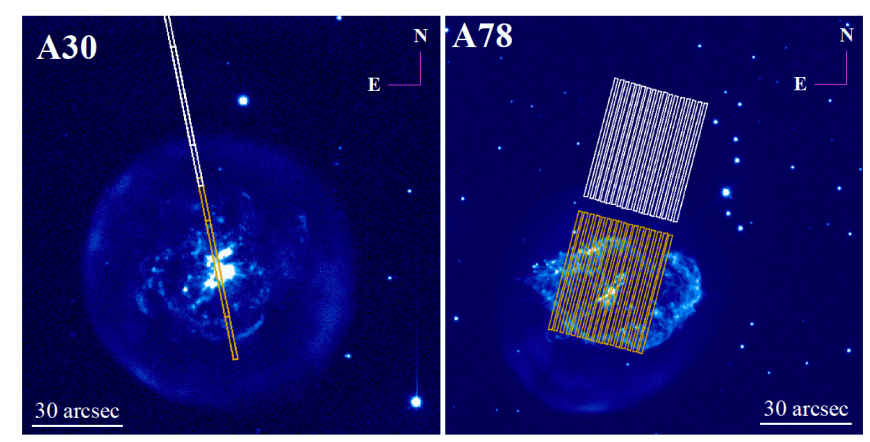

Figure 4. Slit positions of the Spitzer IRS observations of A 30 and A 78. The orange rectangular regions show the positions of the short-low (SL) slits and the white rectangles those used for background subtraction.

$\gtrsim 40^{\prime \prime}$ from the CSPNe with the brightest region spatially coincident with the disrupted disk observed in optical images (see Fang et al. 2014, and references therein). In the case of A 30, a bipolar structure with $\mathrm{PA}=-30^{\circ}$ can be easily distinguished ${ }^{3}$. This is aligned with the bipolar jet seen in optical wavelengths, with NW and SE jet components located 6.' 8 and 7.' 8 from the star, but it seems to extend further out. The lower spatial resolution WISE images of A 78 hinder a similar assessment.

Colour-composite images combining the IR images of A 30 and A 78 with those of their optical [O III] and soft $(0.3-0.5 \mathrm{keV}) \mathrm{X}$-ray $X M M-N e w t o n$ images are presented in the right panels of Figure 1. These pictures show that the hot dust is not only spatially coincident with the hydrogen-poor knots and filaments, but also coexists with the X-ray-emitting material (Guerrero et al. 2012; Toalá et al. 2015).

Figures 2 and 3 present near-IR images of the central regions of A 30 and A 78, respectively, in comparison with their corresponding $H S T$ [O III] images. The NOTCam $J$-band images show only emission from the CSPNe and background stars, although there is the marginal detection of extended emission in A 78. Otherwise, the $H$ - and $K$ band images clearly detect the emission from the disrupted disks of A 30 and A 78. The jets are undetected in the near-IR in agreement with previous reports (e.g., Dinerstein \& Lester 1984; Borkowski et al. 1994), but they are detected in the Spitzer IRAC images of A 30 at longer wavelengths (see Fig. 2 bottom panels). An inspection of the images shows that the jet-like features extend 15.9" towards the NW and $18.2^{\prime \prime}$ to the SE from the CSPN. Their tips are coincident with hydrogen-deficient clumps identified in Figure 2 top left panel which are aligned with the knots identified as the NW and SE jet components. We note that the A $78 \mathrm{~K}$-band image is suggestive of emission located around the location of the jets (see Fig. 3 top right panel).

Figures 2 and 3 also show that the clumps in the disrupted disks of A 30 and A 78 do not present uniform surface brightness. As for A 30, the SW clumps are more IR bright than other clumps, whereas in A 78 the brightest knots are located SE of the CSPN. This spatial distribution is similar to that presented in the [O III] HST images, as well as in the high spatial resolution Chandra X-ray image of A 30, which shows an X-ray emission peak SW of the CSPN (see figure 6 in Guerrero et al. 2012).

\footnotetext{
3 The zero point of the PAs is defined by the north $\left(\mathrm{PA}=0^{\circ}\right)$ and increase
} counterclockwise.

\section{SPECTRAL PROPERTIES OF DUST IN A 30 AND A 78}

Figure 4 shows the slit positions of the low-resolution Spitzer IRS observations of A 30 and A 78. The background was selected from slits without any contribution from the $\mathrm{H}$-deficient knots (shown as white slits of Figure 4). The resultant background-subtracted spectra are shown in Figure 5.

The Spitzer IRS spectra of A30 and A78 are almost identical, which reflects the similar evolutionary stage and born-again origin discussed in previous works (e.g., Fang et al. 2014; Toalá et al. 2015). The low-resolution IRS spectra of A 30 and A 78 presented in the top left panel of Figure 5 show a number of broad features, which were thoroughly discussed by García-Hernández et al. (2013). Those authors compared the spectra of A 30 and A 78 with those of RCBs. After an appropriate continuum subtraction technique, GarcíaHernández et al. (2013) demonstrated that RCB objects exhibited the presence of broad features at 5.9, 6.4, 7.3 and $8.0 \mu \mathrm{m}$ whilst the bornagain PNe only present the latter three broad features (see Figure 13 in García-Hernández et al. 2013). These authors concluded that the presence of such broad features can be attributed to amorphous carbon formed in H-deficient environments. Although subtle differences can be appreciated between A 30 and A 78, which can be attributed to the signal-to-noise in the spectra, both sources exhibit the broad features with the 6.4 and $8.0 \mu \mathrm{m}$ as the strongest and clearest features in Figure 5 top left panel.

The IRS observations of A 78 performed in mapping mode offer us the possibility to create spectral maps. Spectral maps of the 6.4 and $8.0 \mu \mathrm{m}$ broad features are shown in Figure 6. We note that we also attempted to create images of the other broad features, but they resulted in low-quality images due to their lower signal-to-noise. The images of the 6.4 and $8.0 \mu \mathrm{m}$ broad features in A 78 show that these carbon-rich bumps are mainly associated with the disk. Furthermore, the spectra of A 78 show that the CanariCam images presented in the bottom panels of Figure 3 are not due to emission lines, but to dust continuum.

The bottom panel of Figure 5 presents the high-resolution IRS spectra. The spectra do not present a large number of emission lines with the most prominent ones being those of [Ne III] $15.55 \mu \mathrm{m}$ and [O Iv] $25.9 \mu \mathrm{m}$. Other less intense lines are those of [Ne v] 14.32 $\mu \mathrm{m},[\mathrm{Ne} \mathrm{II}] 12.81 \mu \mathrm{m}$ and [Fe v] $34.12 \mu \mathrm{m}$. Some absorption lines are also easily identified in the high-resolution spectra. In particular, we would like to note the $\mathrm{N} v 29.43 \mu \mathrm{m}$ absorption line which reflects the presence of gas with temperatures of $\sim 10^{5} \mathrm{~K}$, very likely the mixing region between the hot bubble $\left(\sim 10^{6} \mathrm{~K}\right)$ and the nebular gas $\left(10^{4} \mathrm{~K}\right)$ (see Fang et al. 2016, and references therein). The $\mathrm{N} v$ absorption line is detected with signal-to-noise larger than 30 for the three born-again PNe (see Appendix A).

For comparison with A 30 and A 78 we also show in Figure 5 the corresponding low- and high-resolution IRS spectra of the younger born-again PN A 58. The IRS spectra of A 58 are more intense for wavelengths below $15 \mu \mathrm{m}$ and is below those of A 30 and A 78 for longer wavelengths. The high-resolution spectrum does not show the higher ionization $\mathrm{Ne}$ lines, but it exhibits the [Ne II] $12.81 \mu \mathrm{m}$ line consistent with the lower $T_{\text {eff }}$ of its CSPN compared to those of A 30 and A 78. It also shows several absorption features very likely due to molecular material present in the born-again ejecta of A 58, similarly to what has been described for Sakurai's Object (see Evans et al. 2006, 2020). We would like to note that the low-resolution spectrum of A 58 also exhibits hints of the $8 \mu \mathrm{m}$ broad feature better appreciated in the top right panel of Figure 5.

Finally, we present in the top right panel of Figure 5 the available IR 

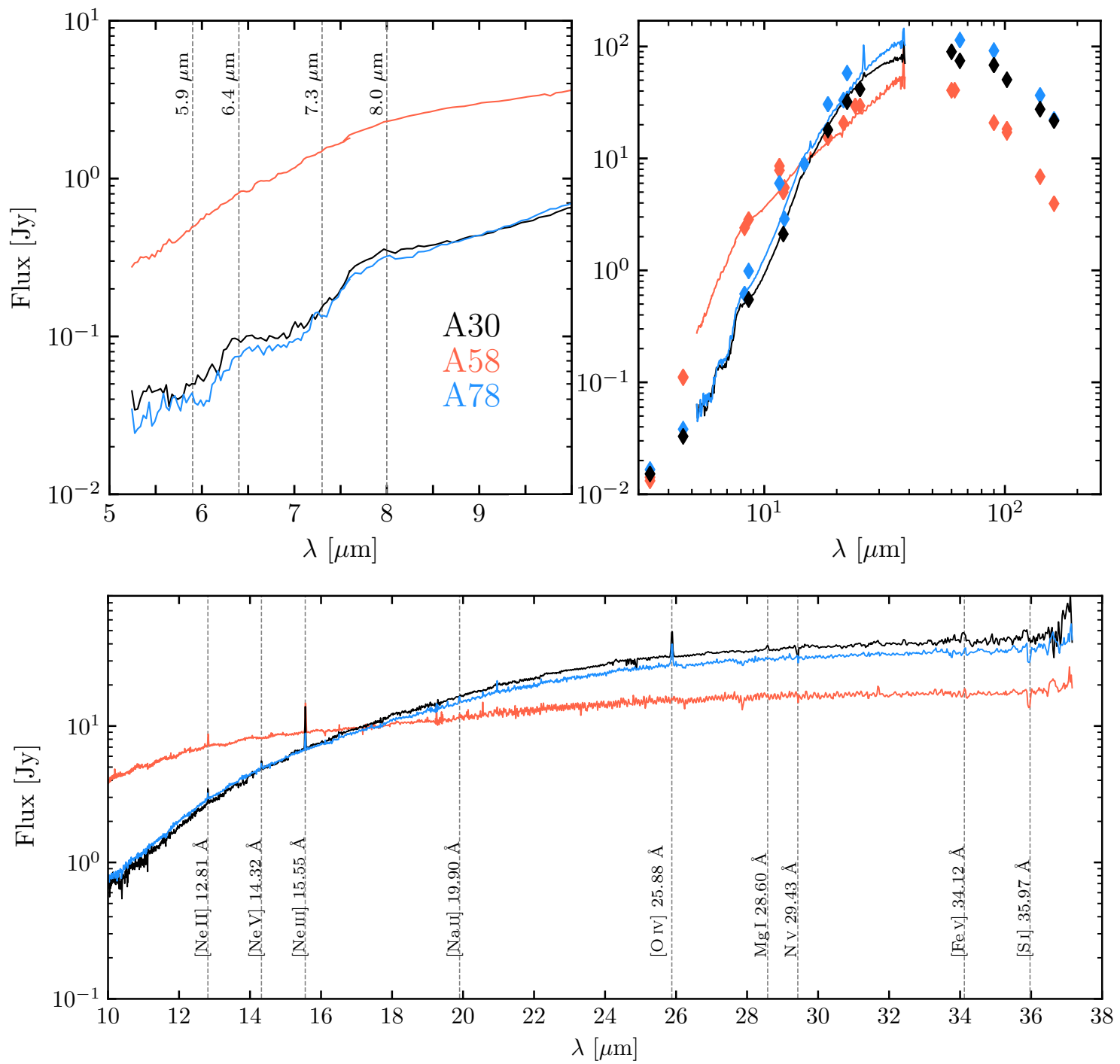

Figure 5. Spitzer IRS spectra of A 30, A 58 and A 78. Top panels: low-resolution IRS spectra. The top-left panel shows the 5-10 $\mu \mathrm{m}$ range with the central wavelengths of the amorphous carbon features found by García-Hernández et al. (2013) shown with vertical dashed lines at 6.4, 7.3 and 8.0 $\mu$ m. The top-right panel shows the complete wavelength range with the corresponding photometry plotted with diamonds for each born-again PN. Bottom: High-resolution IRS spectra. The most prominent atomic and ionic lines are marked.

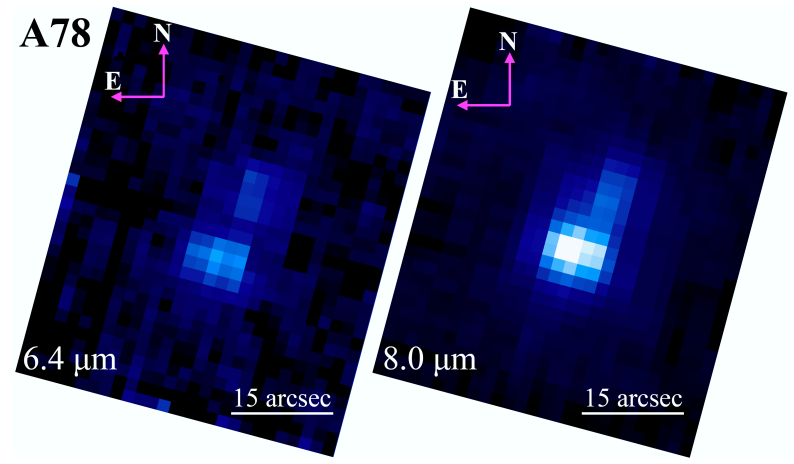

Figure 6. Spitzer IRS spectral maps of A 78 extracted at the 6.4 (left panel) and $8.0 \mu \mathrm{m}$ (right panel) broad spectral features. The pixel size of the IRS maps is $1 . " 8$. photometry for A 30, A 58 and A 78 obtained from the NASA/IPAC IR Science Archive.

\section{DUST MODELLING}

Our observations have shown that the IR properties of A 30 and A 78 are spatially and spectroscopically extremely similar. In addition, previous multi-wavelength studies have shown that these two bornagain PNe share practically all their nebular characteristics and stellar atmosphere analysis of their CSPNe have also shown that they are twins (see Guerrero et al. 2012; Toalá et al. 2015). In this section we will present a dust model tailored to A 30 adopting a distance of 


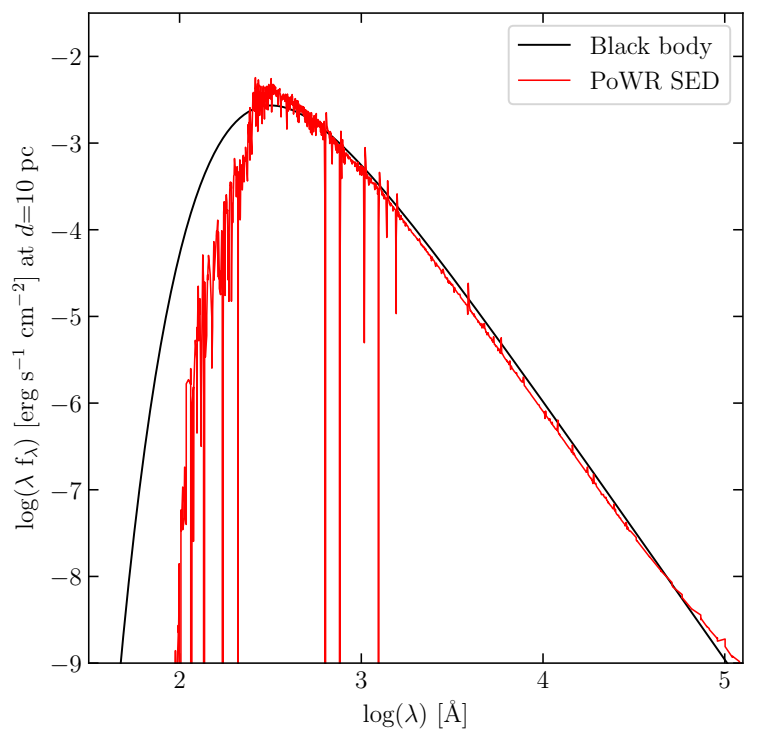

Figure 7. Comparison between the best-fit model to the stellar atmosphere of A 30 obtained with the PoWR code and a black body emission model with a temperature of $115 \mathrm{kK}$.

$d=2.4 \mathrm{kpc}$ as estimated by Bailer-Jones et al. (2018) using astrometric observations from Gaia 4 .

Recent high-quality works have demonstrated that an appropriate description of dust in nebulae around evolved stars must also account for the behavior of gas in the calculations (see, e.g., Gómez-Llanos et al. 2018; Rubio et al. 2020; Jiménez-Hernández et al. 2020). Thus, in order to peer into the dust properties of A 30 and A 78 we have used the photoionization code CLOUdy (version 17.0; Ferland et al. 2017), which models simultaneously the emission of ionized gas and dust as a consequence of the presence of a radiation field. By coupling the PyCLoudy libraries (Morisset 2013) we are able to produce synthetic observations to be directly compared to the real ones.

CLOUDY requires several user-defined inputs: i) the incident spectrum, ii) the chemical abundances, iii) the density distribution and geometry, and iv) the dust physical properties (size and chemical composition) and spatial distribution. Our group has modeled the optical and UV observations of the CSPN of A 30 using the state-ofthe-art stellar atmosphere code PoWR (Gräfener et al. 2002; Hamann \& Gräfener 2004) ${ }^{5}$. The best model for the stellar atmosphere of the CSPN of A 30 has $\log \left(L / \mathrm{L}_{\odot}\right)=3.78$ and $T_{\text {eff }}=115 \mathrm{kK}$ (see the details of the model in Guerrero et al. 2012). The synthetic stellar atmosphere model obtained from PoWR will be used in CLOUDY as the incident spectrum. This is compared to a black body emission model with the same temperature in Figure 7, revealing the noticeable opacity of the dense atmosphere of Wolf-Rayet-type stars to hard photons.

There are not many studies of the physical properties and chemical abundances of the hydrogen-deficient clumps in A 30 in the literature

\footnotetext{
4 A subsequent paper addressing the dust and gas properties of A 78, which is located at $1.5 \mathrm{kpc}$, using high-quality spectroscopic observations will be presented shortly (Montoro-Molina et al. in prep.)

5 http://www.astro.physik. uni-potsdam.de/ wrh/PoWR/

powrgrid1.php
}

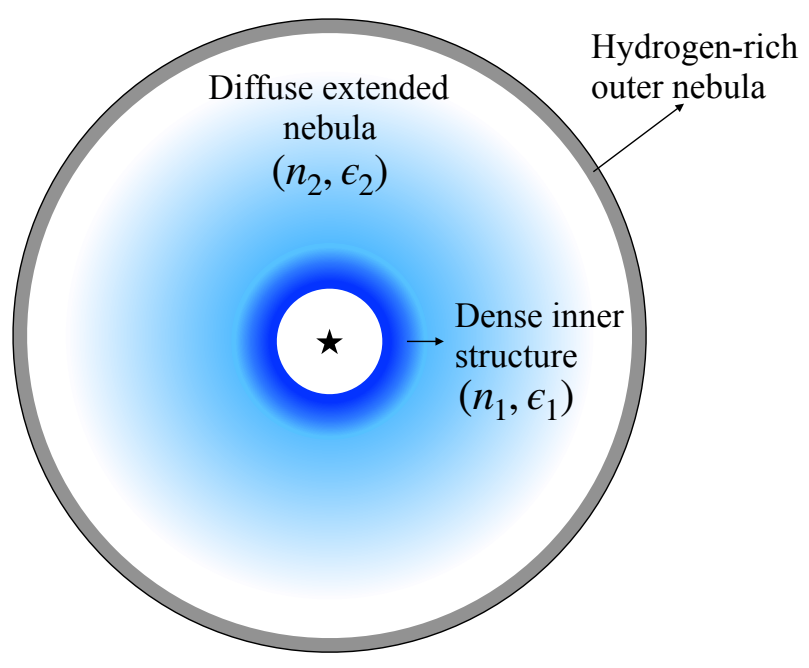

Figure 8. Two-shell distribution model adopted in our CLOUDY models.

(Jacoby \& Ford 1983; Guerrero \& Manchado 1996; Wesson et al. 2003). Here we will adopt the $\mathrm{He}, \mathrm{O}, \mathrm{N}$ and $\mathrm{Ne}$ abundances of knot 1 determined by Guerrero \& Manchado (1996) complemented with the $\mathrm{C} / \mathrm{O}=0.3$ by mass reported by Wesson et al. (2003). Other elemental abundances were set to their solar values (Wilms et al. 2000).

For comparison with previous models of the dust in A 30, we started by taking into account two structures to model the nebular and IR properties of this born-again PN. A dense shell structure close to the CSPN with inner and outer radii of $r_{\text {in }}=8.6^{\prime \prime}$ and $r_{\text {mid }}=15^{\prime \prime}$, respectively. These radii have been obtained by averaging the dense, hydrogen-deficient clumps distances to the CSPN of A 30. A secondary shell structure was defined with an inner radius of $r_{\text {mid }}=15^{\prime \prime}$ and an outer radius $r_{\text {out }}$ which will be kept as a free parameter in our model, but a maximum limit of $60^{\prime \prime}$ is predefined by the optical radius of the hydrogen-rich PN. The total number densities and filling factors of the two shells are denoted as $n_{1}, n_{2}, \epsilon_{1}$ and $\epsilon_{2}$, respectively. For both shells we adopted density distributions with a dependence with radius. For example, for the inner shell we define its density distribution as

$n(r)=n_{1}\left(\frac{r}{r_{1}}\right)^{-\alpha}$,

where $n_{1}$ is the total number density of the gas at its inner face located at $r_{1}$. A similar equation is defined for the outer shell. Figure 8 presents a schematic view of this configuration.

CLOUDY give us the possibility to include in our calculations different built-in dust species (silicates and carbonaceous; Ferland et al. 2017). According to the evolution of the born-again event, we will include carbon-rich dust. We will present a model including amorphous carbon, but we will also compare with models including graphite $^{6}$ and fluffy grains (porous aggregates of smaller grains). Borkowski et al. (1994) suggested that a dust size distribution with a slope of -3 described better the IR SED of A 30 (in contrast to

\footnotetext{
6 We note that Borkowski et al. (1994) rejected the presence of graphite in A 30, however, laboratory experiments have demonstrated the graphitization of amorphous carbon material by the presence of a radiation field (see, e.g., Ogmen \& Duley 1988; Kim, Lee \& Lee 2016, and references therein).
} 

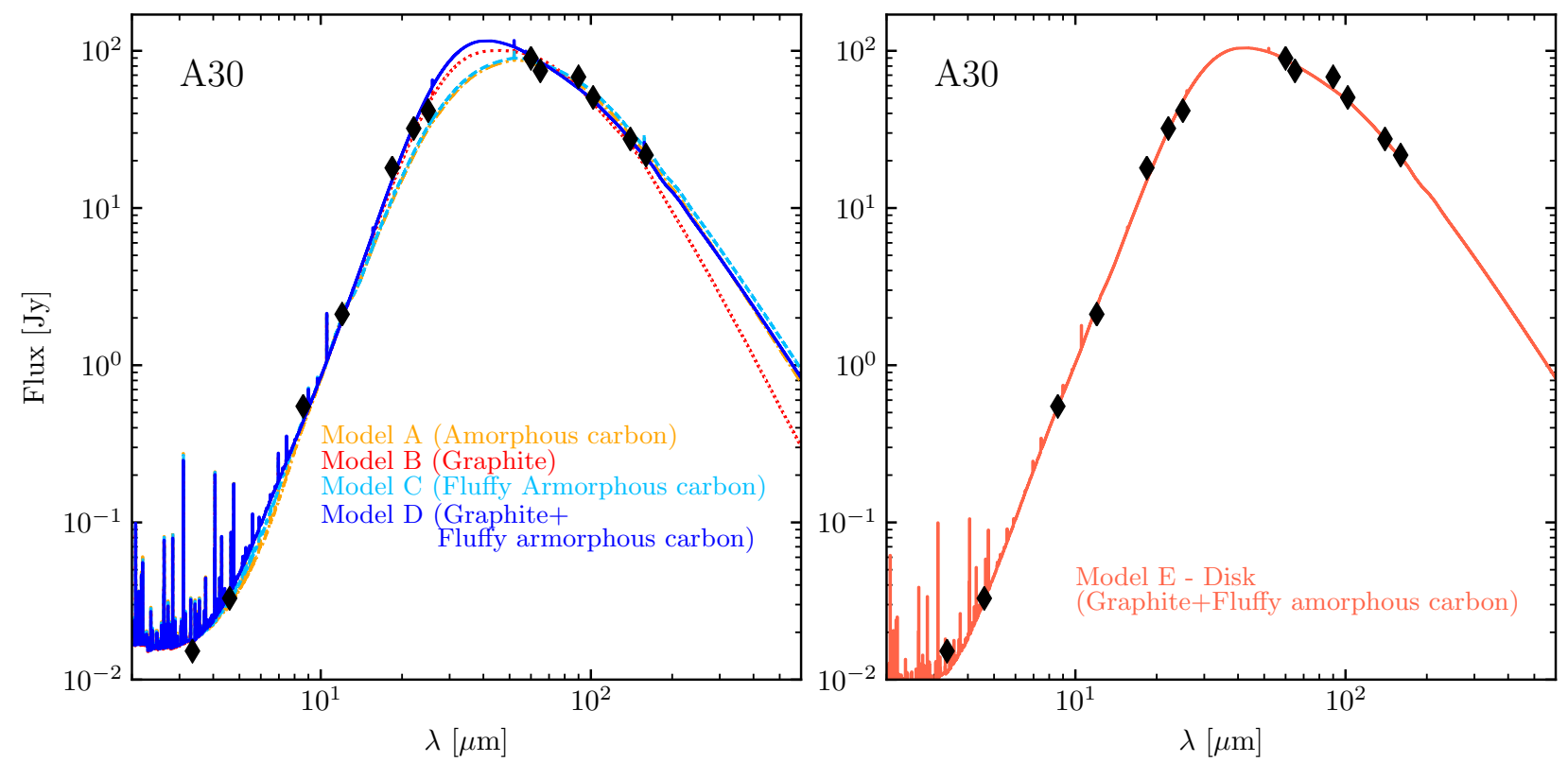

Figure 9. Comparison between the observed IR SED of A 30 (black diamonds) with our CLoudy models. Different colours and line styles shown the spectrum from models as labeled on the panel. The right panel shows our best model taking into account a disk morphology for the inner region.

the $a^{-3.5}$ suggested by Mathis et al. 1977, for the ISM). Following these authors, we fixed the slope of the dust size distribution to -3 in all the following calculations, but we change the limiting values of the dust sizes in different models. We start our model by using grain sizes between 0.0007 and $0.25 \mu \mathrm{m}$ with a power-law distribution of radii of $\propto a^{-3}$. However, in order to improve our models we had to define dust populations with small $\left(a_{\text {small }}\right)$ and big sizes $\left(a_{\text {big }}\right)$. Each distribution of sizes is divided into 10 bins.

Once the model is computed, synthetic nebular observations will be obtained using PyCLOUDY for a slit 1.5 arcsec in width and 3.7 arcmin in length, similar to the one used in Guerrero \& Manchado (1996).

\subsection{Model exploration}

We remark that CLOUDY not only takes into account the contribution from dust, but also computes the properties of the gaseous component. That is, the 1-5 $\mu \mathrm{m}$ wavelength range includes not only the contribution from small grains (as discusses in Borkowski et al. 1994), but also the contribution from emission lines and continua from the ionized gas (see Fig. 9). The combination of the density and small grain size distribution need to be accounted for simultaneously. However, one can restrict the contribution from the ionized gas by trying to fit the $\mathrm{H} \alpha$ flux and the electron temperature $T_{\mathrm{e}}\left(\left[\mathrm{O}_{\text {III }}\right]\right)$ estimated using the [O III] emission lines. Guerrero \& Manchado (1996) reported $\log (\mathrm{H} \alpha) \approx-15$ and $T_{\mathrm{e}}\left(\left[\mathrm{O}_{\mathrm{III}}\right]\right)=14,000 \mathrm{~K}$, whilst Wesson et al. (2003) report $T_{\mathrm{e}}\left(\left[\mathrm{O}_{\mathrm{III}}\right]\right) \lesssim 18,000 \mathrm{~K}$ using different emission lines. We found that for the inner shell $n_{0}=2100 \mathrm{~cm}^{-3}$ and $\epsilon=7.7 \times 10^{-4}$ closely reproduce the $\mathrm{H} \alpha$ flux and electron temperatures. This electron density agrees with that reported in Guerrero \& Manchado (1996) for knot $1\left(2100 \mathrm{~cm}^{-3}\right)$. Thus, for the four models described in the following we fixed $n_{0}$.

We started by adopting amorphous carbon with sizes between 0.0007 and $0.25 \mu \mathrm{m}$ prescribed by Borkowski et al. (1994), but no successful fit could be achieved. In short, dust of these sizes pro- duce considerable emission in the $1-5 \mu$ m that exceeds the observed emission of A 30. This simple exercise showed that grains as small as $0.0007 \mu \mathrm{m}$ cannot be used to reproduce the IR properties of A 30 in conjunction with its optical properties.

In order to appropriately model the SED of A 30 including dust and gas, we need to fit the size range of our dust distribution. Our first model, Model A, required two population of amorphous carbon grains, a population of small grains with sizes of $0.0008-0.03 \mu \mathrm{m}$ present only in the inner shell and larger grains with sizes between $0.04-0.10 \mu \mathrm{m}$ present only in the outer region. Details of the model are listed in Table 1. Model A predicts a total dust mass of $M_{\text {dust, } \mathrm{A}}=$ $6.34 \times 10^{-3} \mathrm{M}_{\odot}$, with $99.4 \%$ corresponding to dust in the outer shell. The total predicted mass of gas, which is computed from the $\mathrm{H} \alpha$ flux, is $M_{\text {gas, }}=1.16 \times 10^{-2} \mathrm{M}_{\odot}$ with $87 \%$ of this retained in the inner shell. Model A is compared to the observed SED in the left panel of Figure 9 (yellow line). This figure shows that Model A does a good job fitting the IR SED of A 30 with a predicted peak at 50-60 $\mu \mathrm{m}$, but there is certain deviation from the photometry for wavelengths between 15 and $30 \mu \mathrm{m}$. Gómez-Llanos et al. (2018) discussed a similar problem for the normal H- and C-rich PN IC 418 and attribute it to the presence of hydrogenated carbonaceous species not included in CLOUDY (see Discussion section). However, we note that the specific cases of A 30 and A 78 studied here are different as no hydrogenated dust species are expected (see previous section).

A second model was performed with the same density distribution but including only graphite in both shells, Model B. This model required that the size ranges of both the smaller and larger grains be shifted to larger values, of $0.001-0.03 \mu \mathrm{m}$ and much bigger large grains of $0.30-0.60 \mu \mathrm{m}$. Model B resulted in a larger total dust mass of $M_{\text {dust,B }}=1.98 \times 10^{-2} \mathrm{M}_{\odot}$. Around $99 \%$ of the total dust is also required to be present in the outer shell, similarly to Model A. Model B is shown as a red line in Figure 9 left panel. This model is better than Model A fitting the IR SED around 15-30 $\mu \mathrm{m}$, but some emission is lacking at longer wavelengths. Furthermore, its SED peak appears displaced towards shorter wavelengths, peaking 
Table 1. Details of our CLOUDY models of the nebular and dust in born-again PNe.

\begin{tabular}{|c|c|c|c|c|c|}
\hline Parameter & $\begin{array}{l}\text { Model A } \\
\text { (Amorphous } \\
\text { carbon) }\end{array}$ & $\begin{array}{l}\text { Model B } \\
\text { (Graphite) }\end{array}$ & $\begin{array}{c}\text { Model C } \\
\text { (Fluffy amorphous } \\
\text { carbon) }\end{array}$ & $\begin{array}{c}\text { Model D } \\
\text { (Graphphite+Fluffy } \\
\text { amorphous carbon) }\end{array}$ & $\begin{array}{c}\text { Model E } \\
\text { (Graphite+Fluffy } \\
\text { amorphous carbon) }\end{array}$ \\
\hline Inner structure & shell & shell & shell & shell & disk \\
\hline$n_{1}\left(\mathrm{~cm}^{-3}\right)$ & 2100 & 2100 & 2100 & 2100 & 2100 \\
\hline$\alpha$ & 3 & 3 & 3 & 3 & 3 \\
\hline$\epsilon\left(\times 10^{-4}\right)$ & 7.7 & 7.7 & 7.7 & 7.7 & 7.7 \\
\hline$r_{\text {in }}(")$ & 8.6 & 8.6 & 8.6 & 8.6 & 8.6 \\
\hline$r_{\text {mid }}\left({ }^{\prime \prime}\right)$ & 15 & 15 & 15 & 15 & 15 \\
\hline Amorphous carbon size distribution & $0.0008-0.03$ & $\ldots$ & $0.0011-0.02$ & $\ldots$ & $0.0011-0.02$ \\
\hline Graphite size distribution & $\ldots$ & $0.001-0.03$ & $\ldots$ & $0.001-0.03$ & $0.001-0.03$ \\
\hline $\mathrm{M}_{\text {gas }}\left(\times 10^{-3} \mathrm{M}_{\odot}\right)$ & 10.06 & 10.06 & 10.06 & 10.06 & 2.85 \\
\hline $\mathrm{M}_{\text {dust }}\left(\times 10^{-3} \mathrm{M}_{\odot}\right)$ & 0.036 & 0.23 & 0.029 & 0.23 & 0.10 \\
\hline gas-to-dust & 278 & 45 & 353 & 45 & 25 \\
\hline \multicolumn{6}{|l|}{ Outer Shell } \\
\hline$n_{2}\left(\mathrm{~cm}^{-3}\right)$ & 55 & 55 & 55 & 55 & 55 \\
\hline$\alpha$ & 3 & 3 & 3 & 3 & 3 \\
\hline$\epsilon\left(\times 10^{-4}\right)$ & 4 & 4 & 4 & 4 & 4 \\
\hline$r_{\text {mid }}\left({ }^{\prime \prime}\right)$ & 15 & 15 & 15 & 15 & 15 \\
\hline$r_{\text {out }}\left({ }^{\prime \prime}\right)$ & 50 & 50 & 50 & 50 & 50 \\
\hline Amorphous carbon size distribution & $0.04-0.10$ & $\ldots$ & $0.025-0.10$ & $0.06-0.20$ & $0.06-0.20$ \\
\hline Graphite size distribution & $\ldots$ & $0.30-0.60$ & $\ldots$ & $\ldots$ & $\ldots$ \\
\hline $\mathrm{M}_{\text {gas }}\left(\times 10^{-3} \mathrm{M}_{\odot}\right)$ & 1.56 & 1.56 & 1.56 & 1.56 & 1.56 \\
\hline $\mathrm{M}_{\text {dust }}\left(\times 10^{-3} \mathrm{M}_{\odot}\right)$ & 6.30 & 19.6 & 2.94 & 3.10 & 3.10 \\
\hline Gas-to-dust & 0.25 & 0.07 & 0.53 & 0.50 & 0.50 \\
\hline$M_{\mathrm{TOT}, \text { gas }}\left(\times 10^{-3} \mathrm{M}_{\odot}\right)$ & 11.62 & 11.62 & 11.62 & 11.62 & 4.41 \\
\hline$M_{\mathrm{TOT}, \text { dust }}\left(\times 10^{-3} \mathrm{M}_{\odot}\right)$ & 6.34 & 19.83 & 2.97 & 3.32 & 3.20 \\
\hline $\log \left(F_{\mathrm{H} \alpha}\right)\left(\mathrm{erg} \mathrm{cm}^{-2} \mathrm{~s}^{-1}\right)$ & -15.03 & -15.06 & -15.04 & -15.06 & -15.09 \\
\hline$T_{\mathrm{e}}([\mathrm{O} \mathrm{III}])(\mathrm{K})$ & 21,400 & 16,600 & 22,400 & 18,400 & 18,500 \\
\hline \multicolumn{6}{|l|}{ Observed } \\
\hline $\log (F(\mathrm{H} \alpha))$ & $-15.04 \pm 0.09$ & & & & \\
\hline$T_{\mathrm{e}}\left(\left[\mathrm{O}_{\mathrm{III}}\right]\right)(\mathrm{K})$ & $14,000-18,000$ & & & & \\
\hline
\end{tabular}

at $\sim 40 \mu \mathrm{m}$, when compared to Model A. Details of the model are listed in Table 1 labeled as Model B.

Model C is similar to Model A but adopting fluffy amorphous carbon grains. We used CLOUDY to construct distributions of amorphous carbon with empty fractions of $50 \%$. To produce a good fit to the IR $\mathrm{SED}$, this model required dust populations of small and big grans with sizes of $0.0011-0.02 \mu \mathrm{m}$ and $0.025-0.10 \mu \mathrm{m}$, respectively. The total dust mass resulted in $M_{\text {dust,C }}=2.97 \times 10^{-3} \mathrm{M}_{\odot}$ with $99 \%$ of the dust in the outer shell. Figure 9 left panel shows that the synthetic SED of Model C is very similar to that of Model A although it resulted in only $15 \%$ of the total dust mass because these lighter fluffy grains have higher absorption cross-sections. Similarly to Model A, Model C peaks between 50-60 $\mu \mathrm{m}$ and has trouble fitting the SED around $15-30 \mu \mathrm{m}$.

Model D was performed taking into account a combination of graphite and fluffy amorphous carbon dust. This models required dust populations of small and big grains of $0.001-0.03 \mu \mathrm{m}$ and $0.06-$ $0.20 \mu \mathrm{m}$, respectively, with total dust mass of $M_{\text {dust, } \mathrm{D}}=3.32 \times$ $10^{-3} \mathrm{M}_{\odot}$. This model requires $93 \%$ of the total dust mass to be present in the outer shell. Model D does a better job than all previously presented models, fitting simultaneously most of the photometric measurements presented in Figure 9.

Following our findings the model that best reproduces the observed IR SED of A 30 in the mid- and far-IR includes the contribution from fluffy amorphous carbon and graphite (Model D). In order to create a more realistic model of the distribution of dust and gas in A 30, we redefined the inner structure of the model. Instead of adopting a spherical shell, we now adopted a disk (torus) structure. The inner and outer radii are taken to be the same as for the inner shell in previous models, 8.6 and 15 arcsec, respectively, with a thickness of $6.4 \mathrm{arcsec}$. All of the parameters of the outer shell are the same as those of Model D, however, we now include a combination of graphite and fluffy amorphous carbon in the inner disk structure and only fluffy amorphous carbon in the outer shell. Details of this model are listed in Table 1 as Model E.

Model E resulted in total mass of gas $M_{\text {gas, }}=\left(4.41_{-0.14}^{+0.55}\right) \times$ $10^{-3} \mathrm{M}_{\odot}$, with $65 \%$ of the total mass in the inner disk. We note that the errors in the estimated ionized mass were now computed taking into account the errors in the $\mathrm{H} \alpha$ flux (see bottom rows of Table 1). The total mass of dust resulted in $M_{\text {dust,E }}=3.20 \times 10^{-3} \mathrm{M}_{\odot}$ with $95 \%$ of the dust to be in the outer shell. This model is compared to the observed SED in the right panel of Figure 9. As a result of the smaller mass of the disk compared to a shell, the contribution of the emission lines in the 1-5 $\mu \mathrm{m}$ has diminished compared to the other models. We would like to note that whereas the mass of the gas is constrained by the $F(\mathrm{H} \alpha)$, the total mass of dust depends strongly on how empty the fluffy grains are. We reckon that an empty fraction of $50 \%$ is an arbitrary value and thus, computed a range of models covering different fractions. Taking into account fluffy amorphous carbon grains with 0 and $90 \%$ empty fractions we estimate that the 

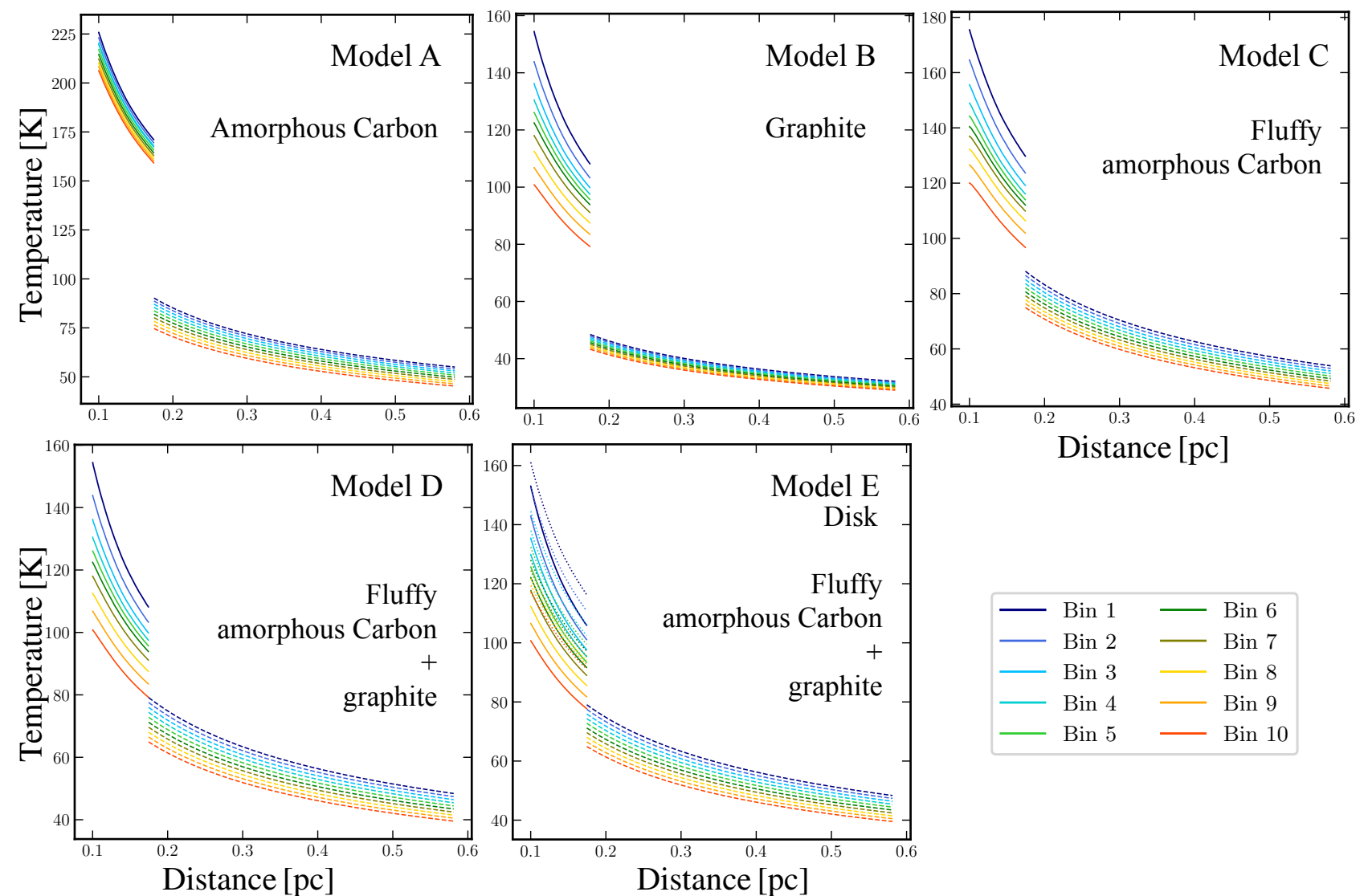

Distance $[\mathrm{pc}]$

Figure 10. Temperature distribution of the different dust grain sizes obtained from the models presented in Section 5 (see Table 1) as a function of distance to the CSPN. Each colour represents different bins in the dust size distributions. The solid (dashed) lines show the temperature of the small (large) grains. Note the differences in temperature scale between all panels. In particular, in the Model E panel the dotted lines show the temperature for the graphite grains in the inner disk.

dust mass of a disk model is $M_{\text {dust, } \mathrm{E}}=\left(3.20_{-2.06}^{+3.21}\right) \times 10^{-3} \mathrm{M}_{\odot}$, which still reproduce the IR SED.

In Figure 10 we present the temperature distribution of the 5 models presented in this section as a function of distance from the CSPN. All of our models predict dust temperatures in the 50-230 K range. In particular, Model A accounting only for amorphous carbon has the highest dust temperatures in the inner shell with $T_{\text {dust }}=150$ $225 \mathrm{~K}$. In all models, the large grains have temperatures between 40 and $100 \mathrm{~K}$.

\section{DISCUSSION}

Previous IR studies of A 30 and A 78 did not have the spatial resolution nor the FoV necessary to unveil the extension of the dust in these two born-again PNe. The analysis of mid-IR Spitzer IRAC and WISE images have shown that dust is present (at least) to radii $\sim 40^{\prime \prime}$. The collection of IR images presented here have shown that the disrupted disk is brighter at shorter wavelengths while the surrounding clumps and filaments increase their brightness towards longer wavelengths, suggesting that small hotter grains are located close to the CSPNe.

The Spitzer IRS observations of A 30 and A 78 presented here exhibit the first imaging evidence of amorphous carbon in these PNe. The broad features below $10 \mu \mathrm{m}$ correspond to amorphous carbon formed in H-deficient environments (e.g., García-Hernández et al.
2013) and are mapped with the spectral mapping capabilities of the Spitzer IRS observations of A 78, mainly located in the inner disk. This situation is interesting and impacts even the discussion of the X-ray emission in born-again PNe. Guerrero et al. (2012) and Toalá et al. (2015) found that the temperature of the X-ray-emitting gas within A 30 and A 78 are the softest among PNe. Although hydrodynamic mixing and/or thermal conduction is usually invoked to explain soft X-ray temperatures, born-again PNe are extremely soft sources $\left(T_{\mathrm{X}} \lesssim 10^{6} \mathrm{~K}\right)$. Guerrero et al. (2012) found that the maximum in the $\mathrm{X}$-ray emission is coincident with the brightest H-deficient clump detected in the [O $\mathrm{III}] H S T$ images. Accordingly, they suggested that charge exchange reaction processes could be occurring on the surface of the C-rich dust (see Dennerl 2010; Dennerl et al. 1997) helping explaining the dominant $\mathrm{C}$ IV emission line in the X-ray spectra. We suggest that heavy ions from the stellar wind of the CSPN of A 30 and $\mathrm{A} 78$ take electrons from the neutral amorphous carbon material and produce the soft X-ray emission.

To our knowledge, there is no further evidence of other molecules in A 30 and A 78 at IR wavelengths nor sub-millimeter observations, although we note that complex molecules, such as hydrogenated and nitrogenated amorphous carbon (among others), have been reported to be present in Sakurai's Object (see Clayton \& De Marco 1997; Evans et al. 2006, 2020; Eyres et al. 1998; Tafoya et al. 2017; Pavlenko et al. 2004) and CO has been detected in A 58 (Tafoya et al. 2017).

The analysis presented here suggests that modeling the IR SED 
of A 30 and A 78 with purely amorphous carbon is a simplification and that other carbonaceous dust species must be included in the calculations. Following previous studies of the IR emission of bornagain PNe, we started our CLOUDY models adopting dust composed only by amorphous carbon. Small grain sizes as those suggested by Borkowski et al. (1994) produce an excess in the near-IR when including the contribution of gas. UV photons ionize the gas and heat up the dust grains, that is, the resultant properties of the IR emission are different as in the absence of the former.

The purely amorphous carbon case (Model A) with a small grain size of $0.0008 \mu \mathrm{m}$ has problems in fitting the IR SED between 15$30 \mu \mathrm{m}$. As mentioned before, Gómez-Llanos et al. (2018) modeled the IR properties of the H- and C-rich PN IC 418 and argued that the emission in this range could be due to unknown carbon-rich hydrogenated species currently not included in CLOUdy. For example, Kwok \& Zhang (2011) showed that some broad features in this wavelength range can be explained by the presence of amorphous organic solids with a mixed aromatic-aliphatic structures and Grishko et al. (2001) showed that hydrogenated amorphous carbon produces broad features in the 20-30 $\mu$ m wavelength range. However, the specific case of born-again $\mathrm{PNe}$ would require amorphous carbonaceous solids with little or no $\mathrm{H}$. The inclusion of graphite (pure carbon solids) in the inner disk can help us addressing this issue.

Previous estimates of the dust temperature in born-again PNe differed considerably depending on the IR range modeled, with dust temperatures of $1000 \mathrm{~K}$ for near-IR $\sim 2-3 \mu$ m measurements (Cohen et al. 1977), but around $145 \mathrm{~K}$ with a marginal decrease of temperature with distance from the CSPN for mid-IR 10 and $20 \mu \mathrm{m}$ observations (Dinerstein \& Lester 1984). We demonstrated here that an agreement is only possible if the contribution from the ionized gas is considered. Our test models resulted in dust temperatures between 40 and $225 \mathrm{~K}$, with different ranges depending on the dust species included in the calculation, but a clear gradient of declining temperature towards the outer regions (see Fig. 10). Our disk model (Model E) required small grains with temperatures in the $\sim 80-160 \mathrm{~K}$ and large grains in the $40-80 \mathrm{~K}$ temperature range. If one takes into account the dust temperatures estimated for Sakurai's Object (1000 K) and A 58 (200-800 K), our results imply that the dust temperature decreases with time, with the dust in the most evolved born-again PNe (A 30 and A 78) having the lowest temperature.

Our best model that reproduces the nebular and IR properties of A 30, Model E, considers an inner disk-like distribution of matter composed of graphite and fluffy amorphous carbon grains. It predicts a total mass of dust of $M_{\text {dust }}=\left(3.20_{-2.06}^{+3.21}\right) \times 10^{-3} \mathrm{M}_{\odot}$, which is very similar to the values of $[2-5] \times 10^{-3} \mathrm{M}_{\odot}$ reported in previous works (see Moseley 1980; Borkowski et al. 1994). We reckon that modeling the amorphous carbon dust with fluffy grains might be more realistic compared to the usually adopted solid spheres. Amorphous carbon dust are structured in chain-like distributions (Rotundi et al. 1998), whilst the optical and IR properties of graphite are less sensitive to shape (see Rai \& Botet 2017). However, the exact empty fraction is unknown and limits the capability of the models to determine a more accurate $M_{\text {dust }}$. To illustrate this, the range of dust mass presented here has been computed by a adopting $0 \%$ and $90 \%$ empty fractions for the amorphous carbon particles. Emptier dust particles include less dust mass but are still able to reproduce the IR SED because their absorption cross-sections are higher.

The total mass of gas predicted by Model E is $M_{\text {gas }}=\left(4.41_{-0.14}^{+0.55}\right) \times$ $10^{-3} \mathrm{M}_{\odot}$. Then, the total mass ejected during the VLTP can be estimated to be

$M_{\mathrm{VLTP}}=M_{\mathrm{dust}}+M_{\mathrm{gas}}=\left(7.61_{-2.20}^{+3.76}\right) \times 10^{-3} \mathrm{M}_{\odot}$.
If the born-again event lasted 20-100 yr, as predicted by stellar evolution models (see Miller Bertolami et al. 2006), the mass-loss rate during the VLTP would be $\dot{M}_{\mathrm{VLTP}} \approx[5-60] \times 10^{-5} \mathrm{M}_{\odot} \mathrm{yr}^{-1}$, although due to the clumpy nature of the amorphous carbon dust, we favor the lower limits of $M_{\mathrm{VLTP}}$ and $\dot{M}_{\mathrm{VLTP}}$. Even then, this value is at the top of the reported mass-loss rates of AGB stars in the literature (see Ramstedt et al. 2020, and references therein), but we note that the origin of the VLTP is explosive. This is the first time that an estimation of the mass lost during the VLTP is attempted for A 30. Interestingly, Guerrero et al. (2018) presented stellar evolution models to try to unveil the evolutive path of the born-again PNe $\mathrm{HuBi} 1$ and found that a mass-loss rate during the born-again event of $7.6 \times 10^{-5} \mathrm{M}_{\odot} \mathrm{yr}^{-1}$ reproduces best their observations. However the total ejected mass predicted by Guerrero et al. (2018) is $8 \times 10^{-4} \mathrm{M}_{\odot}$.

All of our models predict that most of the mass in dust is present in the outer shell, which is located between the inner disrupted disk and the outer relic, H-rich nebula. The reasons for this situation might be a combination of physical effects. Radiation pressure over the grains will cause them to be accelerated pushing them away from the CSPN and the complex hydrodynamic processes occurring in the H-deficient clumps and filaments of A 30 and A 78 (see discussion section in Fang et al. 2014) might drag the dust along with the gas depending on the coupling degree. However, one might also need to take into account the destruction of dust close to the CSPN (see Borkowski et al. 1994). Within $1000 \mathrm{yr}$ after the born-again event in A 30 and A 78 it is difficult to assess which effect has dominated over the other. This could be only explored by future radiation-hydrodynamic simulations that include dust physics and a detailed evolution of the stellar wind parameters and UV flux from stellar evolution models tailored to VLTP events.

\subsection{The born-again versus the nova-like scenario}

The born-again scenario described in Section 1 has been challenged by the nebular abundance measurements of their H-deficient material. Using optical spectroscopic observations Wesson et al. (2003, 2008) estimated that the $\mathrm{C} / \mathrm{O}$ ratio by mass of the $\mathrm{H}$-poor material in A 30 and A 58 have values between 0.06 and $\lesssim 0.3$, whilst the born-again scenario calculations predict $\mathrm{C} / \mathrm{O} \gtrsim 1$ (see table 2 in Miller Bertolami et al. 2006). Following this discrepancy, Lau et al. (2011) suggested that born-again $\mathrm{PNe}$ were better explained by nova-like eruptions, which predict C/O values around 0.05-0.60 (see, e.g., Starrfield et al. 1998), instead of the VLTP scenario. However, modeling the optical and IR observations of A 30 with CLOUDY we have demonstrated that around 40 per cent of $M_{\mathrm{VLTP}}$ might have coagulated into C-rich dust, which is not accounted for in the nebular $\mathrm{C} / \mathrm{O}$ estimations described above.

Using the nebular abundances by mass fraction of $\mathrm{C}$ and $\mathrm{O}$ reported for knot 1 in A 30 by Wesson et al. (2003) (see also table 1 in Lau et al. 2011), that is $X_{C}=0.07$ and $X_{O}=0.27$, we can estimate their contribution to the gas as

$M_{\text {gas, } \mathrm{C}}=X_{\mathrm{C}} \times M_{\text {gas }}=3.1 \times 10^{-4} \mathrm{M}_{\odot}$

and

$M_{\text {gas, } \mathrm{O}}=X_{\mathrm{O}} \times M_{\text {gas }}=1.2 \times 10^{-3} \mathrm{M}_{\odot}$,

respectively. On the other hand, the mass of carbon in the dust is exactly the dust mass determined by our best model, $M_{\text {dust }}$. This follows from the fact that amorphous carbon and graphite are allotropic forms of carbon, that is, they are formed only of carbon. Finally, we 
can estimate the $\mathrm{C} / \mathrm{O}$ mass ratio as

$\mathrm{C} / \mathrm{O}=\frac{M_{\text {gas, } \mathrm{C}}+M_{\text {dust }}}{M_{\text {gas }, \mathrm{O}}} \geqslant 1.27$,

the later adopting the lower limit to the dust mass.

The revised value of the $\mathrm{C} / \mathrm{O}$ ratio is then consistent with the bornagain scenario predictions instead of a nova-like event. The possible role of the binary companion of the CSPN of A 30 in the ejection of the $\mathrm{H}$-poor material is then downgraded, although it might still have helped shaping the axisymmetric ejecta.

\section{SUMMARY}

We presented the analysis of IR observations of the born-again PNe A 30 and A 78. These two PNe have experienced an explosive event that ejected processed material inside their old nebular structures. Previous optical observations have shown that, in both cases, the $\mathrm{H}$ deficient material is distributed in a disk and jet close to their CSPN plus a collection of clumps and filaments distributed more or less homogeneously inside their old, hydrogen-rich PNe.

IR images and spectra covering the $3-160 \mu \mathrm{m}$ wavelength range were used in conjunction with the photoionization code CLOUDY to study the distribution of carbon-rich dust in A 30 and A 78 and to characterize its properties. Our findings can be summarized as:

- We confirm previous suggestions that the dust in A 30 and A 78 is not only distributed in a disk close to the star. Mid-IR images obtained from Spitzer IRAC and WISE show that dust is present up to distances $\sim 40$ arcsec from their CSPN. Comparison with optical and X-ray images show that the dust is spatially correlated with the $\mathrm{H}$-deficient clumps (including the jet features) and filaments inside the old $\mathrm{H}$-rich $\mathrm{PNe}$, and that it coexists with the recently-powered X-ray-emitting hot bubbles $\left(T_{\mathrm{X}} \lesssim 10^{6} \mathrm{~K}\right)$.

- Analysis of the near- and mid-IR images suggest that the dust present in the disks of A 30 and A 78 have different properties than their jet features. In particular, comparison between $H$ and $K$ band images of A 30 with those obtained with Spitzer IRAC show that the jet starts to be detected at wavelengths longer than $3 \mu \mathrm{m}$.

- The spectral maps obtained with the Spitzer IRS observations show the first imaging evidence of amorphous carbon in A 78. These are mapped through the 6.4 and $8.0 \mu \mathrm{m}$ broad spectral features. Its spatial coincidence with the $\mathrm{X}$-ray emission supports the charge exchange reactions at the surface of these carbon-rich structures as the mechanism for the production of super soft X-ray in born-again PNe. We detect the N v $29.43 \AA$ line in absorption in A 30, A 58 and A 78, which has been suggested to originate in the $10^{5} \mathrm{~K}$ gas resulted from the mix of the X-ray-emitting gas and the ionized material. Thus, we predict that as well as the other two objects, A 58 might have started to develop a hot bubble.

- A variety of CLOUDY models including a number of dust species were used to study their contribution in different regions of the IR SED. Models with single dust species are not able to reproduce simultaneously the SED in the mid- and far-IR. A combination of different carbon-rich species need to be taken into account, for example, our best model includes the contribution from fluffy amorphous carbon and graphite. The advantage of using CLOUDY is that we can simultaneously fit the nebular and dust properties for a more complete description of born-again $\mathrm{PNe}$ and produce improved estimates of dust temperatures. The dust temperatures predicted by our models are in the $40-230 \mathrm{~K}$ range, in stark contrast with previous estimates of $1000 \mathrm{~K}$.
- Our best model for the nebular and IR properties of A 30 was obtained by adopting a disk structure near the CSPN with the contribution of an outer spherical region, gas and dust is included in both regions. The total estimated gas is $\left(4.41_{-0.14}^{+0.55}\right) \times 10^{-3} \mathrm{M}_{\odot}$ with a very similar total mass of dust of $\left(3.20_{-2.06}^{+3.21}\right) \times 10^{-3} \mathrm{M}_{\odot}$. The model requires a small fraction $<5 \%$ of the dust to be present in the disk with only the contribution from small grains $(0.001-0.03 \mu \mathrm{m})$. Most dust is in the outer shell, specifically the big grains $(0.06-$ $0.20 \mu \mathrm{m})$. This situation shows the complex interactions dragging the dust away from the CSPN which might involve radiation pressure, hydrodynamic processes and dust destruction.

- If the total mass ejected in the VLTP of A 30 is $M_{\text {VLTP }}=$ $M_{\text {dust }}+M_{\text {gas }}=\left[7.61_{-2.20}^{+3.76}\right] \times 10^{-3} \mathrm{M}_{\odot}$, then we estimated a massloss rate of $\dot{M}_{\text {VLTP }} \approx[5-60] \times 10^{-5} \mathrm{M}_{\odot} \mathrm{yr}^{-1}$. Due to the very likely porous (chain-like) structure of amorphous carbon grains, we favor lower values for both $M_{\mathrm{VLTP}}$ and $\dot{M}_{\mathrm{VLTP}}$, which are otherwise consistent with previous estimates for the born-again PN HuBi 1.

- We have shown that the $\mathrm{C}$ mass in the H-poor ejecta of A 30 is larger than the $\mathrm{O}$ mass once that the $\mathrm{C}$ atoms trapped into dust are taken into account. This result is consistent with the predictions of the born-again scenario and contradicts the expectations of a nova-like event suggested previously.

Born-again PNe are complex entities with only a few cases known which remain to be unveiled. Future SOFIA HAWC+ observations at longer wavelengths could be used to constrain the contribution from graphite in evolved born-again PNe. Similar studies of other known born-again $\mathrm{PNe}$ will be pursued to assess the $\mathrm{C} / \mathrm{O}$ mass ratio discrepancy pointed out in previous works. Future radiation-hydrodynamic numerical simulations including dust-related physics, following in detail the evolution of the CSPN, might be able to shed light into the complex phenomena occurring in born-again $\mathrm{PNe}$.

\section{ACKNOWLEDGEMENTS}

The authors are thankful to the anonymous referee for a detailed report of our manuscript which improved its presentation and clarity. The authors thank O. González-Martín (IRyA-UNAM) for reducing the CanariCam data. The authors thank funding by Dirección General de Asuntos del Personal Académico (DGAPA) of the Universidad Nacional Autónoma de México (UNAM) project IA100720. JAT thanks Fundación Marcos Moshinsky (Mexico). VMAGG acknowledges support from the Programa de Becas posdoctorales funded by DGAPA UNAM. JBRG, SED and PJH thank Consejo Nacional de Ciencias y Tecnología (CONACyT) México for research student grants. MAG acknowledges support of the Spanish Ministerio de Ciencia, Innovación y Universidades (MCIU) grant PGC2018102184-B-I00. GRL acknowledges support from CONACyT (grant 263373) and PRODEP (Mexico). DAGH acknowledges support from the State Research Agency (AEI) of the Spanish Ministry of Science, Innovation and Universities (MCIU) and the European Regional Development Fund (FEDER) under grant AYA2017-88254-P.

Based on observations made with the instruments NOTCam at the Nordic Optical Telescope (NOT) and CanariCam at the Gran Telescopio Canarias (GTC), installed in the Spanish Observatorio del Roque de los Muchachos of the Instituto de Astrofísica de Canarias, in the island of La Palma. NOT is owned in collaboration by the University of Turku and Aarhus University, and operated jointly by Aarhus University, the University of Turku and the University of Oslo, representing Denmark, Finland and Norway, the University of Iceland and Stockholm University. This work makes use of Spitzer 
IR observations which was operated by the Jet Propulsion Laboratory, California Institute of Technology under a contract with NASA. Support for this work was provided by NASA through an award issued by JPL/Caltech. This work is partially based on observations obtained with XMM-Newton, an ESA science mission with instruments and contributions directly funded by ESA Member States and NASA. This work has made extensive use of NASA's Astrophysics Data System.

\section{DATA AVAILABILITY}

The data underlying this article will be shared on reasonable request to the corresponding author.

\section{REFERENCES}

Asplund, M., Gustafsson, B., Lambert, D. L., \& Kameswara Rao, N. 1997, A\&A, 321, L17

Bailer-Jones, C. A. L., Rybizki, J., Fouesneau, M., et al. 2018, AJ, 156, 58 Borkowski, K. J., Harrington, J. P., \& Tsvetanov, Z. I. 1995, ApJ, 449, L143 Borkowski, K. J., Harrington, J. P., Blair, W. P., et al. 1994, ApJ, 435, 722 Borkowski, K. J., Harrington, J. P., Tsvetanov, Z., et al. 1993, ApJ, 415, L47 Clayton, G. C., Bond, H. E., Long, L. A., et al. 2013, ApJ, 771, 130

Clayton, G. C., Kerber, F., Pirzkal, N., et al. 2006, ApJ, 646, L69

Clayton, G. C. \& De Marco, O. 1997, AJ, 114, 2679

Cohen, M., \& Barlow, M. J. 1974, ApJ, 193, 401

Cohen, M., Hudson, H. S., Odell, S. L., et al. 1977, MNRAS, 181, 233

Dennerl, K. 2010, Space Sci. Rev., 157, 57

Dennerl, K., Englhauser, J., \& Trümper, J. 1997, Science, 277, 1625

Dinerstein, H. L., \& Lester, D. F. 1984, ApJ, 281, 702

Duerbeck, H. W., Pollacco, D., Verbunt, F., et al. 1996, IAU Circ., 6328, 1

Duerbeck, H. W., Liller, W., Sterken, C., et al. 2000, AJ, 119, 2360

Evans, A., Gehrz, R. D., Woodward, C. E., et al. 2020, MNRAS, 493, 1277

Evans, A., Tyne, V. H., van Loon, J. T., et al. 2006, MNRAS, 373, L75

Eyres, S. P. S., Evans, A., Geballe, T. R., et al. 1998, MNRAS, 298, L37

Fang, X., Guerrero, M. A., Toalá, J. A., et al. 2016, ApJ, 822, L19

Fang, X., Guerrero, M. A., Marquez-Lugo, R. A., et al. 2014, ApJ, 797, 100

Ferland, G. J., Chatzikos, M., Guzmán, F., et al. 2017, Rev. Mex. Astron. Astrofis., 53, 385

García-Hernández, D. A., Rao, N. K., \& Lambert, D. L. 2013, ApJ, 773, 107

Gómez-Llanos, V., Morisset, C., Szczerba, R., et al. 2018, A\&A, 617, A85

González-Martín, O., Rodríguez-Espinosa, J. M., Díaz-Santos, T., et al. 2013, A\&A, 553, A35

Gräfener, G., Koesterke, L., \& Hamann, W.-R. 2002, A\&A, 387, 244

Grishko, V. I., Tereszchuk, K., Duley, W. W., et al. 2001, ApJ, 558, L129

Gruendl, R. A., Chu, Y.-H., \& Guerrero, M. A. 2004, ApJ, 617, L127

Guerrero, M. A., Fang, X., Miller Bertolami, M. M., et al. 2018, Nature Astronomy, 2, 784

Guerrero, M. A., Ruiz, N., Hamann, W.-R., et al. 2012, ApJ, 755, 129

Guerrero, M. A., \& De Marco, O. 2013, A\&A, 553, A126

Guerrero, M. A., \& Manchado, A. 1996, ApJ, 472, 711

Gvaramadze, V. V., Kniazev, A. Y., Gräfener, G., et al. 2020, MNRAS, 492, 3316

Hamann, W.-R., \& Gräfener, G. 2004, A\&A, 427, 697

Herwig, F., Blöcker, T., Langer, N., \& Driebe, T. 1999, A\&A, 349, L5

Hinkle, K. H., \& Joyce, R. R. 2015, Why Galaxies Care about AGB Stars III: A Closer Look in Space and Time, 497, 303

Hinkle, K. H., Lebzelter, T., Joyce, R. R., et al. 2008, A\&A, 479, 817

Iben, I., Kaler, J. B., Truran, J. W., et al. 1983, ApJ, 264, 605

Iping, R. C., Sonneborn, G., \& Chu, Y.-H. 2002, American Astronomical Society Meeting Abstracts

Jacoby, G. H., Hillwig, T. C., \& Jones, D. 2020, MNRAS, 498, L114

Jacoby, G. H., \& Ford, H. C. 1983, ApJ, 266, 298

Jacoby, G. H. 1979, PASP, 91, 754
Jiménez-Hernández, P., Arthur, S. J., \& Toalá, J. A. 2020, MNRAS, 497, 4128

Kim, T., Lee, J., \& Lee, K.-H., 2016, RSC Adv., 6, 24667

Koller, J. \& Kimeswenger, S. 2001, ApJ, 559, 419

Kwok, S. \& Zhang, Y. 2011, Nature, 479, 80

Lau, H. H. B., De Marco, O., \& Liu, X.-W. 2011, MNRAS, 410, 1870

Leene, A. \& Pottasch, S. R. 1988, A\&A, 202, 203

Mathis, J. S., Rumpl, W., \& Nordsieck, K. H. 1977, ApJ, 217, 425

Meaburn, J., Lopez, J. A., Bryce, M., et al. 1998, A\&A, 334, 670

Miller Bertolami, M. M., Althaus, L. G., Serenelli, A. M., \& Panei, J. A. 2006, A\&A, 449, 313

Morisset, C. 2013, pyCloudy: Tools to manage astronomical Cloudy photoionization code, ascl:1304.020

Moseley, H. 1980, ApJ, 238, 892

Muthumariappan, C. \& Parthasarathy, M. 2020, MNRAS, 493, 730

Nakano, S., Sakurai, Y., Hazen, M., et al. 1996, IAU Circ., 6322, 1

Ogmen, M. \& Duley, W. W. 1988, ApJ, 334, L117

Pavlenko, Y. V., Geballe, T. R., Evans, A., et al. 2004, A\&A, 417, L39

Pollacco, D. 1999, MNRAS, 304, 127

Pottasch, S. R., Baud, B., Beintema, D., et al. 1984, A\&A, 138, 10

Rai, R. K. \& Botet, R. 2017, MNRAS, 467, 2009

Ramstedt, S., Vlemmings, W. H. T., Doan, L., et al. 2020, A\&A, 640, A133

Rotundi, A., Rietmeijer, F. J. M., Colangeli, L., et al. 1998, A\&A, 329, 1087

Rubio, G., Toalá, J. A., Jiménez-Hernández, P., et al. 2020, MNRAS, 499, 415

Ruiz, N., Chu, Y.-H., Gruendl, R. A., et al. 2013, ApJ, 767, 35

Schönberner, D. 1979, A\&A, 79, 108

Smith, J. D. T., Armus, L., Dale, D. A., et al. 2007, PASP, 119, 1133

Soker, N. 1994, AJ, 107, 276

Starrfield, S., Truran, J. W., Wiescher, M. C., et al. 1998, MNRAS, 296, 502

Tafoya, D., Toalá, J. A., Vlemmings, W. H. T., et al. 2017, A\&A, 600, A23

Toalá, J. A., Guerrero, M. A., Todt, H., et al. 2015, ApJ, 799, 67

Toalá, J. A. \& Arthur, S. J. 2018, MNRAS, 478, 1218

Toalá, J. A. \& Arthur, S. J. 2011, ApJ, 737, 100

Tody, D. 1993, Astronomical Data Analysis Software and Systems II, 173

van Hoof, P. A. M., Hajduk, M., Zijlstra, A. A., et al. 2007, A\&A, 471, L9

Weaver, R., McCray, R., Castor, J., et al. 1977, ApJ, 218, 377

Weidmann, W. A. \& Gamen, R. 2011, A\&A, 526, A6

Wesson, R., Barlow, M. J., Liu, X.-W., et al. 2008, MNRAS, 383, 1639

Wesson, R., Liu, X.-W., \& Barlow, M. J. 2003, MNRAS, 340, 253

Wilms, J., Allen, A., \& McCray, R. 2000, ApJ, 542, 914

\section{APPENDIX A: THE PRESENCE OF THE MIXING LAYER ON BORN-AGAIN PLANETARY NEBULAE}

The temperature structure of wind-blown bubbles results in a somewhat complex distribution. An X-ray-emitting hot bubble $\left(T_{\mathrm{X}} \gtrsim\right.$ $10^{6} \mathrm{~K}$ ) is formed by the adiabatically-shocked stellar wind that pushes the photionized material $\left(10^{4} \mathrm{~K}\right)$. Numerical simulations have long predicted that, when in contact, these two layers might experience a variety of physical processes. The photoionized material might experience hydrodynamical instabilities breaking into clumps and filaments (e.g., Toalá \& Arthur 2011, 2018, and references therein) that are latter mixed with the hot bubble producing gas at intermediate temperatures. This process can be enhanced if thermal conductivity is taken into account (Soker 1994; Weaver et al. 1977). As a result, a mixing (or conduction) layer with temperature $\sim 10^{5} \mathrm{~K}$ between these two layers will appear.

Conduction layers have been detected in other PNe by means of UV observations. Far Ultraviolet Spectroscopic Explorer (FUSE) observations of the O vi $\lambda \lambda 1032,1038$ doublet have been reported in several PNe (Iping et al. 2002; Gruendl et al. 2004; Ruiz et al. 2013). Fang et al. (2016) used HST STIS observations to disclose the presence of the Nv $1239 \AA$ emission line in the Cat's Eye Nebula in comparison with the X-ray-emitting gas and the nebular layers, 


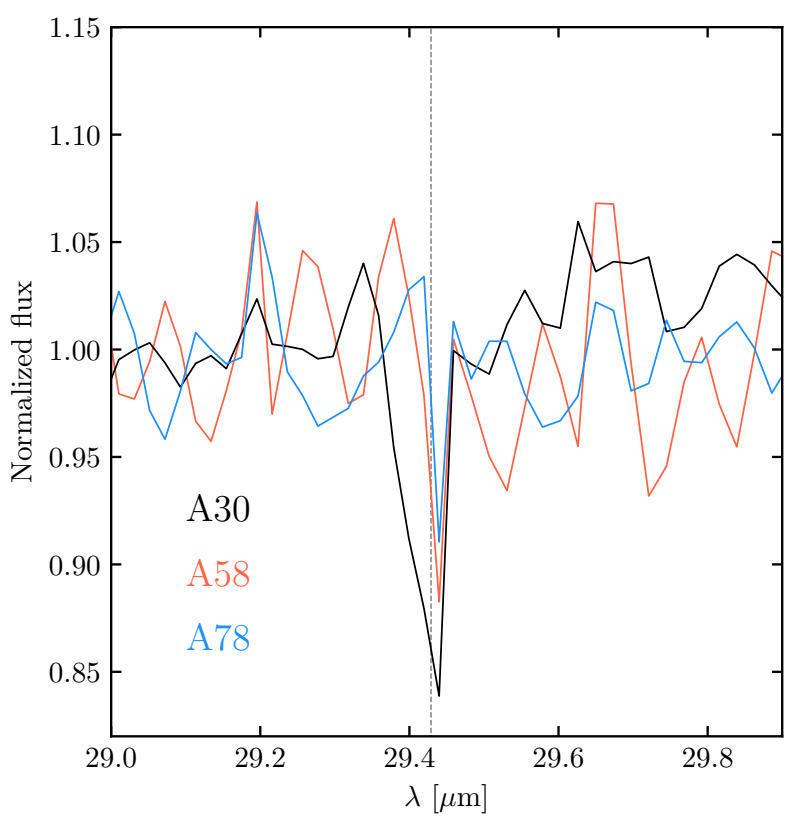

Figure A1. Normalized flux of the high-resolution spectra of A 30, A 58 and A 78 around the $\mathrm{Nv}$ absorption line at $29.43 \mu \mathrm{m}$ (dashed line).

revealing for the first time the distribution of the mixing layer in a PN.

The Nv $29.43 \mu \mathrm{m}$ line is detected in absorption in the highresolution Spitzer IRS observations of the three born-again PNe presented here (see Fig. A1). We used IRAF to estimate that the signal-to-noise ( $\mathrm{S} / \mathrm{N})$ of the $\mathrm{N} v$ line is 54,33 and 38 for A 30, A 58 and A78 with FWHM of $0.06,0.03$ and $0.02 \mu \mathrm{m}$, respectively. These where then compared to the FWHM of the unambiguously detected O IV at $25.88 \mu \mathrm{m}$ which resulted in $0.05 \mu \mathrm{m}$ (A 30), $0.026 \mu \mathrm{m}$ (A 58) and $0.045 \mu \mathrm{m}$ (A 78). Thus, we can conclude that the $\mathrm{N} \mathrm{v}$ absorption line is definitely detected in A 30 and A 58, but questionably in A 78 . However, the reported extended X-ray emission in A 78 corroborates the detection of the mixing layer through the $\mathrm{N} \mathrm{v}$ absorption line.

These results suggest that A 58 might also harbor a hot bubble formed by the interaction of its current fast wind with the dense structure around its CSPN. Future X-ray observations will help assessing this suggestion. 